\title{
Generalized Selection Weighted Vector Filters
}

\author{
Rastislav Lukac \\ The Edward S. Rogers Sr. Department of Electrical and Computer Engineering, University of Toronto, 10 King's College Road, \\ Toronto, ON, Canada M5S $3 G 4$ \\ Email:lukacr@dsp.utoronto.ca
}

Konstantinos N. Plataniotis

The Edward S. Rogers Sr. Department of Electrical and Computer Engineering, University of Toronto, 10 King's College Road, Toronto, ON, Canada M5S $3 G 4$

Email:kostas@dsp.utoronto.ca

\section{Bogdan Smolka}

Department of Automatic Control, Silesian University of Technology, Akademicka 16, 44-101 Gliwice, Poland Email:bsmolka@ia.polsl.gliwice.pl

\section{Anastasios N. Venetsanopoulos}

The Edward S. Rogers Sr. Department of Electrical and Computer Engineering, University of Toronto, 10 King's College Road, Toronto, ON, Canada M5S $3 G 4$

Email:anv@dsp.utoronto.ca

Received 21 July 2003; Revised 11 December 2003

This paper introduces a class of nonlinear multichannel filters capable of removing impulsive noise in color images. The hereproposed generalized selection weighted vector filter class constitutes a powerful filtering framework for multichannel signal processing. Previously defined multichannel filters such as vector median filter, basic vector directional filter, directional-distance filter, weighted vector median filters, and weighted vector directional filters are treated from a global viewpoint using the proposed framework. Robust order-statistic concepts and increased degree of freedom in filter design make the proposed method attractive for a variety of applications. Introduced multichannel sigmoidal adaptation of the filter parameters and its modifications allow to accommodate the filter parameters to varying signal and noise statistics. Simulation studies reported in this paper indicate that the proposed filter class is computationally attractive, yields excellent performance, and is able to preserve fine details and color information while efficiently suppressing impulsive noise. This paper is an extended version of the paper by Lukac et al. presented at the 2003 IEEE-EURASIP Workshop on Nonlinear Signal and Image Processing (NSIP '03) in Grado, Italy.

Keywords and phrases: multichannel image processing, color image processing, nonlinear vector filtering, order-statistic theory, adaptive filter design, noise removal.

\section{INTRODUCTION}

Vector signal processing is of paramount importance in application areas such as biomedicine, computer vision, multimedia, robotics, industrial inspection, and remote sensing. In all these areas, end-users and system developers have to work with multidimensional vectorial data sets. The growing interest in the development of vector processing techniques can be attributed primarily to the importance of color image processing $[1,2]$. The surge of emerging applications [1] such as web-based processing of color images and videos, enhancement of DNA microarray images, digital archiving and culture heritage preservation, multimedia sequence mining, and the proliferation of devices [3] such as video-enabled wireless phones and personal digital assistant (PDA) tools suggests that the demand for new, more powerful, and costeffective vector-filtering solutions will continue.

It is well known that humans and computer vision systems use color information to sense the environment and that the correct perception of color can help in different tasks of image understanding and object recognition. Unfortunately, noise and other impairments associated with the measurement or the transmission apparatus significantly degrade the value of the color information. This usually declines the perceptual quality and the fidelity of the images, and also decreases the performance of the task for which the image was 
used. It comes therefore, as no surprise, that the most common signal processing task is the noise filtering. Noise filtering is an essential part of any image processing-based system, whether the final information is used for human inspection or for an automatic analysis.

In the last decades, several noise reduction techniques have been proposed. They can be divided into two classes: linear filters and nonlinear filters.

Linear processing techniques have been widely used in digital signal processing applications. However, many image processing tasks are suitable for linear operations as well. On the other hand, image signals are nonlinear in nature due to the presence of structural information (edge and flat areas) and are perceived via the human visual system which has strong nonlinear characteristics [4]. Therefore, nonlinear methods can potentially preserve important multichannel structural elements, such as color edges, and eliminate degradations occurring during signal formation or transmission through nonlinear channel.

This paper contributes to the progressive generalization of vector filtering operators. The main emphasis is on the development of a unified framework for the description and analysis of color image filters. Existing, vector color image processing solutions can be discerned as follows:

(i) componentwise (marginal) methods [5, 6], which separately operate on color channels;

(ii) vector (multichannel) methods $[1,2]$, which process the color pixels as vectors.

In the case of red-green-blue (RGB) image representation which is most often used for image acquisition, storing and displaying systems, componentwise filters produce strong, visible color artifacts due to independent (separate) processing of the correlated RGB channels. Their projection into the restored RGB image often produces a new color quite different from the neighbors. Therefore, vector filters, especially nonlinear vector filters, which process the color pixels as vectors seem to be more appropriate $[1,2,7]$.

The most popular class of nonlinear vector operators is the one based on the theory of order statistics $[8,9,10,11]$. In such an approach, the center of the ordered sequence, known as a median [10], represents the sample which has the largest probability to be noise free. In vector-valued signals such as color images, outliers are associated with the maximum extremes of aggregated distances to other input vectors in the sliding window $[12,13,14]$. For that reason, the output of the vector filters based on ranking is the lowest ranked vector in a predefined sliding window. Mostly used vector filtering techniques such as vector median filters (VMFs) [15] and vector directional filters (VDFs) [16] utilize either the aggregated Euclidean distance (in the VMF designs) or the aggregated angular distance (in the VDF designs) of the input vectors within a processing window. However, these measures do not take into account neither the importance of the specific samples in the filter window nor structural contents of the image. Much better results can be obtained when distances appropriately modified by weighting coefficients represent the degree to which each input vec- tor contributes to the output of the filter. The relationship between the image vector at the window center and its neighbors should be reflected in the decision for the weights of the filter.

This paper focuses on a class of nonlinear filters termed selection weighted vector filters (SWVFs) [17]. As the name implies, the output of the filter is selected from an input set associated with the positive weight vector (or two independent weight vectors for Euclidean and angular distances). These weights express the degree of which the specific distances between multichannel inputs contribute to the aggregated measure serving as an ordering criteria. To adapt the filter weights to varying signal and noise statistics, we provide the generalized multichannel adaptation algorithms based on the sigmoidal function [17]. The proposed approach has the advantage of optimal weighted medians (WMs) [18], is fast, saves the memory space and is easy to implement. Moreover, the achieved weights are sufficiently robust and the proposed method is able to remove impulsive noise while preserving edge information.

The rest of this paper is organized as follows. In Section 2, the formulation of the problem is introduced and an overview of the most commonly used vector filters is presented. A generalized framework for vector filtering is introduced in Section 3. Motivation and design characteristics are discussed in detail. Variations of the proposed structure are recommended and analyzed with respect to their properties and parameters used. In Section 4, the proposed methods are tested using a variety of noise corrupted test images. Conclusions are offered in Section 5.

\section{MULTICHANNEL IMAGE FILTERING FUNDAMENTALS}

We consider a $K_{1} \times K_{2}$ multichannel image $\mathbf{x}(i): Z^{l} \rightarrow Z^{m}$ representing a two-dimensional matrix of $m$-component samples $\mathbf{x}_{i}=\left(x_{i 1}, x_{i 2}, \ldots, x_{i m}\right) \in Z^{l}$. Note that standard color images such as RGB images relate to parameters $l$ and $m$ equal to 2 and 3, respectively. Components $x_{i k}$, for $k=1,2, \ldots, m$ and $i=1,2, \ldots, Q ; Q=K_{1} K_{2}$, represent the $k$ th elements of the vectorial inputs $\mathbf{x}_{i}$.

Each multichannel sample $\mathbf{x}_{i}=\left(x_{i 1}, x_{i 2}, \ldots, x_{i m}\right)$ can be considered as an $m$-dimensional vector in the vector space. For a color vector $\mathbf{x}_{i}$, its magnitude $M_{\mathbf{x}}(i): Z^{l} \rightarrow R^{+}$,

$$
M_{\mathbf{x}_{i}}=\left\|\mathbf{x}_{i}\right\|=\sqrt{\left(x_{i 1}\right)^{2}+\left(x_{i 2}\right)^{2}+\cdots+\left(x_{i m}\right)^{2}},
$$

constitutes a measure of their brightness. Its direction $D_{\mathbf{x}}(i)$ : $Z^{l} \rightarrow S^{m-1}$,

$$
\begin{gathered}
D_{\mathbf{x}_{i}}=\frac{1}{\left\|\mathbf{x}_{i}\right\|} \mathbf{x}_{i}=\frac{1}{M_{\mathbf{x}_{i}}} \mathbf{x}_{i}, \\
d_{x_{i k}}=\frac{x_{i k}}{\left\|\mathbf{x}_{i}\right\|}=\frac{x_{i k}}{M_{\mathbf{x}_{i}}}, \quad \text { for } k=1,2, \ldots, m,
\end{gathered}
$$

where $S^{m-1}$ is a unit ball in $R^{m}$ and $\left\|D_{\mathbf{x}_{i}}\right\|=1$, describes their chromaticity. 


\subsection{Problem formulation}

In many practical applications, vectorial signals such as multichannel images are corrupted by additive noise. The most commonly used model is defined $[1,15,19]$ as

$$
\mathbf{x}_{i}=\mathbf{o}_{i}+\mathbf{v}_{i}
$$

where $\mathbf{x}_{i}=\left(x_{i 1}, x_{i 2}, \ldots, x_{i m}\right)$ represents the observed (noisy) sample, $\mathbf{o}_{i}=\left(o_{i 1}, o_{i 2}, \ldots, o_{i m}\right)$ is the desired (noise free) sample, $\mathbf{v}_{i}=\left(v_{i 1}, v_{i 2}, \ldots, v_{i m}\right)$ is the vector describing the noise process (e.g., thermal noise mixed with bit errors), and $i$ denotes the spatial position of the samples in the image. Note that $\mathbf{v}_{i}$ can describe signal-dependent noise as well as noise to be considered independent.

The appearance of the noise and its influence on the image relates to its characteristics. Noise signals can be either periodic in nature or random. In certain cases (e.g., sensors), noise can be described in terms of the commonly used Gaussian noise model. However, noise-corrupted signals are often characterized by abrupt local changes, in which case the noise masking the true signal can be modelled as impulsive sequences, which occur in the form of short time duration, high energy spikes attaining large amplitudes with probability higher than that predicted by a Gaussian density model. Such impulsive noise can be introduced into the images by electronic interference, flaws in the data transmission procedure, or through the aging of the storage material [2].

\subsection{State of the art in color image filtering}

The most popular vector image processing filters, such as the VMF and the VDF, are operating on some type of sliding window $W=\left\{\mathbf{x}_{i} \in Z^{l} ; i=1,2, \ldots, N\right\}$ of a finite size $N$, which usually affects one image sample (mostly the sample $\mathbf{x}_{(N+1) / 2}$ placed in the center of the window) at a time, changing its value by some function of a local neighborhood area $\left\{\mathbf{x}_{1}, \mathbf{x}_{2}, \ldots, \mathbf{x}_{N}\right\}$. This window operator slides over the image to affect individually all the image pixels.

Probably the most well-known filter is the VMF [15]. The VMF can be derived as a maximum likelihood estimate (MLE) when the underlying probability densities of $\mathbf{v}_{i} \in Z^{l}$ are double exponential. The output of the VMF scheme can be equivalently expressed as the sample $\mathbf{x}_{(1)} \in W$, that is, the sample of the input set $W$, minimizing the distance to other samples inside $W$ :

$$
\min \underset{\mathbf{x}_{(1)} \in W}{\arg } \sum_{i=1}^{N}\left\|\mathbf{x}_{(1)}-\mathbf{x}_{i}\right\|_{L}
$$

where $\left\|\mathbf{x}_{i}-\mathbf{x}_{j}\right\|_{L}$ is the generalized Minkowski metric [20] determining the distance between two $m$-channel samples $\mathbf{x}_{i}=\left(x_{i 1}, x_{i 2}, \ldots, x_{i m}\right)$ and $\mathbf{x}_{j}=\left(x_{j 1}, x_{j 2}, \ldots, x_{j m}\right)$,

$$
\left\|\mathbf{x}_{i}-\mathbf{x}_{j}\right\|_{L}=\left(\sum_{k=1}^{m}\left|x_{i k}-x_{j k}\right|^{L}\right)^{1 / L},
$$

where $L$ denotes the norm parameter, for example, the cityblock distance $(L=1)$ or Euclidean distance $(L=2)$, and $x_{i k}$ is the $k$ th element of $\mathbf{x}_{i}$. If the vector dimension $m=1$ (gray-scale case), the VMF is equivalent to the scalar median filter (MF).

Properties of the VMF scheme have been summarized in [15]. One of the most important is the robust noise attenuation capability which makes the VMF very attractive for various applications. However, the drawback of the VMF scheme is its low signal-detail preserving capability, as the VMF tends to produce streaks, that is, regions of constant or nearly constant brightness. This was a motivation for developing of the VMF-based modifications, for example, fast switching schemes [21], weighted filtering structures [22, 23], gradientbased design [24], and combinations with the linear filters [15], rational filters [25], and fuzzy sets [26, 27]. The properties of color spaces have been followed in [28]. To speed up the calculation of the distances between the color vectors, the VMF based on linear approximation of the Euclidean norm has been provided [29].

Another class of vector operators, termed VDF filters [16], is operating on the directional domain of color images. It has been observed [30] that the output of the basic VDF (BVDF) [16] is the color vector $\mathbf{x}_{(1)} \in W$ whose direction is the MLE of directions of the input vectors. Thus, the BVDF output $\mathbf{x}_{(1)}$ minimizes the angular ordering criteria to other samples inside the sliding filtering window $W$ :

$$
\min \underset{\mathbf{x}_{(1)} \in W}{\arg } \sum_{i=1}^{N} A\left(\mathbf{x}_{(1)}, \mathbf{x}_{i}\right),
$$

where

$$
A\left(\mathbf{x}_{i}, \mathbf{x}_{j}\right)=\arccos \left(\frac{\mathbf{x}_{i} \cdot \mathbf{x}_{j}}{\left|\mathbf{x}_{i}\right|\left|\mathbf{x}_{j}\right|}\right)
$$

represents the angle between two $m$-dimensional vectors $\mathbf{x}_{i}$ and $\mathbf{x}_{j}$.

In [30], it has been proven that in the case of color images, the angular minimization approach is useful for directional data such as color data. Therefore, the filtering schemes based on directional processing of color images (or directional processing followed by magnitude processing) $[1,2,16]$ may achieve better performance than the VMFbased approaches in terms of the color chromaticity (direction of color data) preservation. On the other hand, the VDF filters do not take into account the brightness of color vectors. To utilize both features in color image filtering $[2,16]$, the generalized VDFs (GVDFs) and double window GVDF first eliminate the color vectors with atypical directions in the vector space and consequently process the vectors with the most similar orientation according to their magnitude. Thus, the GVDF splits the color image processing into the directional processing and the magnitude processing.

Other approaches such as directional-distance filter (DDF) [31] and hybrid vector filters [32], respectively, combine both ordering criteria used in the VMF and the BVDF schemes and require evaluation of both the VMF and BVDF outputs. Spherical medians (SMFs) [16] minimize the 
angular criteria (6) excluding the constraints of the output sample lying in the filter window $W$. In order to develop robust smoothing algorithms operating on vectors' directionality, fuzzy adaptive filters have been provided $[19,33]$. Recently developed weighted VDF (WVDF) filters $[34,35]$ improve the signal-detail preserving capability of the VDF operators.

\section{PROPOSED FRAMEWORK}

\subsection{Generalization}

Let $W=\mathbf{x}_{1}, \mathbf{x}_{2}, \ldots, \mathbf{x}_{N}$ be a set of vector-valued samples spawned by a filter window of a finite size $N$ and let $\mathbf{x}_{(N+1) / 2}$ be the central sample corresponding to the window reference position. We assume that $\mathbf{w}=\left[w_{1}, w_{2}, \ldots, w_{N}\right]$ and $\mathbf{u}=\left[u_{1}, u_{2}, \ldots, u_{N}\right]$ represent sets of positive real weights, where the weights $w_{i}$ and $u_{i}$, for $i=1,2, \ldots, N$, are associated with the input sample $\mathbf{x}_{i}$.

Applying a minimization procedure similar to the one used for VMF or BVDF, the obtained SWVF output is the sample $\mathbf{x}_{(1)}$ minimizing

$$
\min \underset{\mathbf{x}_{(1)} \in W}{\arg }\left(\sum_{i=1}^{N} w_{i}\left\|\mathbf{x}_{(1)}-\mathbf{x}_{i}\right\|_{L}\right)^{1-p}\left(\sum_{i=1}^{N} u_{i} A\left(\mathbf{x}_{(1)}, \mathbf{x}_{i}\right)\right)^{p},
$$

where $p$ is a design parameter ranged from 0 to 1 . Weight coefficient $w_{i}$ signifies the importance of the input sample $\mathbf{x}_{i}$ based on the aggregated Euclidean distance and $u_{i}$ measures the importance of $\mathbf{x}_{i}$ according to the aggregated angular distance. A design parameter $p$ can be used to tune the overall filter characteristics in terms of color fundamentals [1]. It should be mentioned at this point that the aggregated Euclidean distance relates to the brightness characteristics of the color vectors under consideration, whereas the aggregated angular distance relates to the chromaticity properties of specific samples. Weight coefficients associated with $\mathbf{x}_{i}$ emphasize its importance in (8) by adding the multiplied distances to the aggregated measures of its neighbors. Due to zero self-contribution of $\mathbf{x}_{i}$ to the corresponding aggregated measure, $w_{i}$ and $u_{i}$ make $\mathbf{x}_{i}$ favorable. Thus, the weight coefficients used in the SWVF scheme allow to control its detailpreserving and noise attenuating characteristics.

The output of the SWVF can be determined using the vector order statistics. We denote

$$
\begin{aligned}
\psi_{i}= & \left(\sum_{j=1}^{N} w_{j}\left\|\mathbf{x}_{i}-\mathbf{x}_{j}\right\|_{L}\right)^{1-p} \\
& \times\left(\sum_{j=1}^{N} u_{j} A\left(\mathbf{x}_{i}, \mathbf{x}_{j}\right)\right)^{p}
\end{aligned}
$$

as the combined aggregated measure associated with $\mathbf{x}_{i}$, for $i=1,2, \ldots, N$. Then the ordered sequence of $\psi_{1}, \psi_{2}, \ldots, \psi_{N}$ is given by $\psi_{(1)} \leq \psi_{(2)} \leq \cdots \leq \psi_{(N)}$. Assuming that the ordering of $\psi_{(i)}$ 's implies the same ordering of the input set $\mathbf{x}_{1}, \mathbf{x}_{2}, \ldots, \mathbf{x}_{N}$, the procedure results in the ordered set $\mathbf{x}_{(1)}, \mathbf{x}_{(2)}, \ldots, \mathbf{x}_{(N)}$, where $\mathbf{x}_{(i)}$ is associated with $\psi_{(i)}$. In
TABLE 1: Special cases of the proposed SWVF framework.

\begin{tabular}{lcc}
\hline Filter & SWVF parameters & Reference \\
\hline WVMF & $p=0$ & {$[23]$} \\
WVDF & $p=1$ & {$[35]$} \\
VMF & $w_{i}=1, u_{i}=1, p=0$ & {$[15]$} \\
BVDF & $w_{i}=1, u_{i}=1, p=1$ & {$[16]$} \\
DDF & $w_{i}=1, u_{i}=1$ & {$[31]$} \\
\hline
\end{tabular}

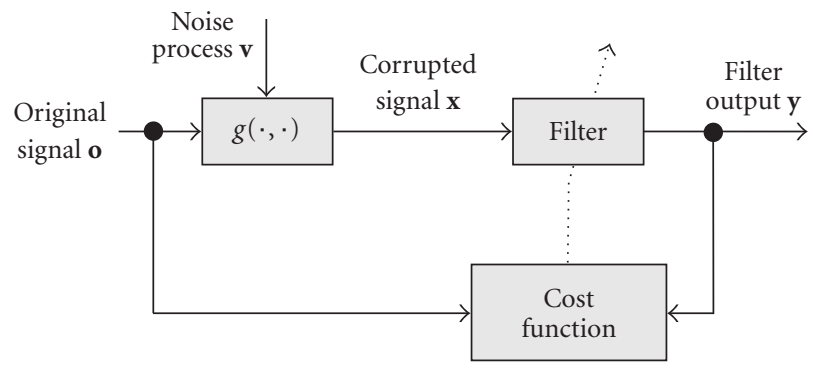

Figure 1: The filtering problem statement, where a filter is usually designed under the minimization of the cost function.

this case, the SWVF output is defined as the lowest orderstatistics $\mathbf{x}_{(1)}$, which is equivalent to the sample-minimizing earlier definition (8). From this algorithm, it is evident that the SWVF output is restricted to the dynamic range of the input samples and thus, it can never cause new outliers.

A class of SWVFs includes (Table 1) a number of previously introduced vector filters as their subclasses. These filters can be achieved by appropriate configuration of the design parameter $p$ and the weight coefficients $w_{1}, w_{2}, \ldots, w_{N}$ and $u_{1}, u_{2}, \ldots, u_{N}$. Thus, the SWVF includes the WVMF (for $p=0$ ) and WVDF (for $p=1$ ) such as basic subclasses. Special cases such as VMF $(p=0), \operatorname{BVDF}(p=1)$, and DDF are obtained with $w_{i}=1, u_{i}=1$, for $i=1,2, \ldots, N$.

\subsection{Adaptive filter design}

SWVFs constitute a wide class of vector operators. Each setting of the filter parameters represents a specific filter which can be used for specific purposes. Using optimization scheme (Figure 1), the weight coefficients can follow the statistics and structural context of desired signal and be adapted in required manner. To simplify the SWVF optimization and provide better illustration of the weight adaptation [17], we consider the equivalence between the weight vectors $\mathbf{w}$ and $\mathbf{u}$ so that $w_{i}=u_{i}$, for $i=1,2, \ldots, N$. Thus, in the rest of this paper, we will make the use of the weight coefficients $w_{1}, w_{2}, \ldots, w_{N}$ only. These coefficients are adaptively determined using generalized multichannel sigmoidal optimization approach [17] of the standard WM filters [18].

The development of vector image processing filters [2] requires the quantification of the distance between the vector-valued samples, and the sample ordering using the obtained distances. Both require a method to compare the vectorial inputs. This can be done by combining the sign operator and the distance measure between two vectors. 
We consider the generalized difference between two vectors $\mathbf{a}$ and $\mathbf{b}$ :

$$
D(\mathbf{a}-\mathbf{b})=S(\mathbf{a}, \mathbf{b})\left(\|\mathbf{a}-\mathbf{b}\|_{L}\right)^{1-p}(A(\mathbf{a}, \mathbf{b}))^{p},
$$

where $S(\cdot) \in\{-1,1\}$ is a polarity function given by

$$
S(\mathbf{a}, \mathbf{b})= \begin{cases}+1, & \text { for }\|\mathbf{a}\|-\|\mathbf{b}\| \geq 0 \\ -1, & \text { for }\|\mathbf{a}\|-\|\mathbf{b}\|<0\end{cases}
$$

Note that the polarity function introduced here preserves the sign of the difference between the scalar image samples $a$ and $b$ since for a scalar case, that is, $m=1$ and $p=0$, the magnitude of $\mathbf{a}$ and $\mathbf{b}$ is equivalent to $a$ and $b$, respectively.

Given an input set $W=\left\{\mathbf{x}_{1}, \mathbf{x}_{2}, \ldots, \mathbf{x}_{N}\right\}$ and a weight vector $\mathbf{w}=\left[w_{1}, w_{2}, \ldots, w_{N}\right]$, we denote the SWVF output as $\mathbf{y}=\mathbf{y}(\mathbf{w}, W)=\mathbf{x}_{(1)}$. The loss in performance (error in the filtering operation) is defined as

$$
e=|D(\mathbf{o}-\mathbf{y})|
$$

Although this function is not convex in nature, it allows to express the error criteria using the same design parameter $p$ like in the SWVF scheme and thus, (12) well describes behavior of the SWVF framework.

One (but no only) natural way of choosing the weight coefficients $w_{1}, w_{2}, \ldots, w_{N}$ is to require that this choice should minimize the average loss or risk. Therefore, the cost function of the SWVF filtering is defined as follows:

$$
J_{\operatorname{SWVF}}(\mathbf{w})=E\{|D(\mathbf{o}-\mathbf{y})|\},
$$

where $E\{\cdot\}$ indicates statistical expectation.

With the constraint of nonnegative weights keeping the aggregated measure (9) positive, the optimization problem with inequality constraints can be expressed as follows:

$$
\begin{gathered}
\text { minimize } J_{\mathrm{SWVF}}(\mathbf{w}) \\
\text { subject to } w_{i} \geq 0 \text { for } i=1,2, \ldots, N .
\end{gathered}
$$

During processing, the sliding filtering window is moving over an image domain. The weight coefficients $w_{i}$, for $i=1,2, \ldots, N$, are adjusted by adding the contribution of the samples multiplied by a certain regulation factor $\mu$ [17]:

$$
w_{i}=P\left[w_{i}+2 \mu D(\mathbf{o}-\mathbf{y}) \operatorname{sgn}\left(D\left(\mathbf{x}_{i}-\mathbf{y}\right)\right)\right],
$$

where $\mathbf{y}$ is the output of the sigmoidally optimized SWVF related to the actual weight coefficients $\mathbf{w}$.

This iterative algorithm determines the weight coefficients with respect to the filter weights at the previous point. If $D(\mathbf{o}-\mathbf{y})$ is zero, a filter holds the preserving characteristics and all weight coefficients remain unchanged. If $D\left(\mathbf{x}_{i}-\mathbf{y}\right)$ is zero, the input sample $\mathbf{x}_{i}$ denotes the same noise attenuation and detail preserving capability as $\mathbf{y}$ and the corresponding weight $w_{i}$ remains also unchanged. In the rest of cases, $D(\mathbf{o}-\mathbf{y})$ and $D\left(\mathbf{x}_{i}-\mathbf{y}\right)$ influence the weight update in terms of the tradeoff between the noise smoothing and the signaldetail preservation. Note that the initial weight vector $\mathbf{w}$ can be set to arbitrary positive values, but the best choice is to start the weight adaptation with equal weights-for example, $w_{i}=1$, for $i=1,2, \ldots, N$-corresponding to the robust smoothing functions such as VMF, BVDF, and DDF. To end the description of $(15)$, notation $\operatorname{sgn}(\cdot)$ denotes the sign sigmoidal function,

$$
\operatorname{sgn}(a)=\frac{2}{1+e^{-a}}-1,
$$

and $P(\cdot)$ is a projection function,

$$
P\left(w_{i}\right)= \begin{cases}0 & \text { if } w_{i}<0 \\ w_{i} & \text { otherwise }\end{cases}
$$

which changes the negative values to zero during the weight adaptation.

For illustration purposes, we consider the scalar case (e.g., componentwise filtering for a particular value of $k$ ). In this case and for $p=0,(15)$ can be written as follows:

$$
w_{i}=P\left[w_{i}+2 \mu\left(o_{k}-y_{k}\right) \operatorname{sgn}\left(x_{i k}-y_{k}\right)\right] \text {. }
$$

Assuming for the moment that $P(\cdot)$ is an identity function, for $x_{i k} \gg y_{k}$ and positive $\mu$, the adaptation formula (18) reduces to

$$
w_{i}=w_{i}+2 \mu\left(o_{k}-y_{k}\right) \text {. }
$$

According to (19), the importance of the sample occupying the $i$ th position in a supporting window increases if $o_{k}$ is greater than the actual output $y_{k}$ and decreases if $o_{k}$ is less than $y_{k}$. Thus, this difference multiplied by a regularization factor represents the weight increment (for $0<o_{k}-y_{k}$ ), the weight decrement (for $0>o_{k}-y_{k}$ ), or it can keep the weights unchanged (for $o_{k}-y_{k}=0$ ).

It is clear that the availability of original (training) signal in (15) is essential in the development of the filter. However, noise-free (training) samples may not be available in practical image processing applications. In such a case, the proposed scheme can be optimized using training sets available from other natural images. Upon completion of the training, the filter can be applied to real images. Another way is to replace the desired signal $\mathbf{o}$ with the input central sample $\mathbf{x}_{(N+1) / 2}$ as follows:

$$
w_{i}=P\left[w_{i}+2 \mu D\left(\mathbf{x}_{(N+1) / 2}-\mathbf{y}\right) \operatorname{sgn}\left(D\left(\mathbf{x}_{i}-\mathbf{y}\right)\right)\right] .
$$

This approach is useful when underlying noise probability is low and detail-preserving characteristics of the SWVFs are required. Another adaptation scheme can be obtained if a robust easily achieved estimate $\mathbf{y}^{*}$, for example, componentwise MF or sample average, is used instead $\mathbf{x}_{(N+1) / 2}$ :

$$
w_{i}=P\left[w_{i}+2 \mu D\left(\mathbf{y}^{*}-\mathbf{y}\right) \operatorname{sgn}\left(D\left(\mathbf{x}_{i}-\mathbf{y}\right)\right)\right] \text {, }
$$

where $\mathbf{y}^{*}=\left(y_{1}^{*}, y_{2}^{*}, \ldots, y_{N}^{*}\right)$ is the MF of the input set $W$ :

$$
y_{k}^{*}=\operatorname{med}\left\{x_{1 k}, x_{2 k}, \ldots, x_{N k}\right\},
$$




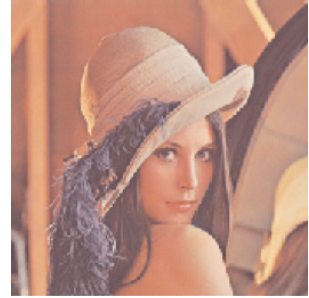

(a)

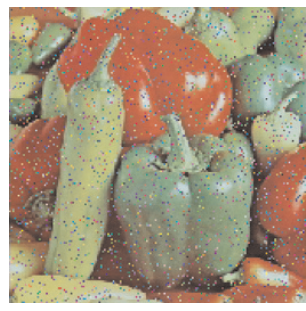

(e)

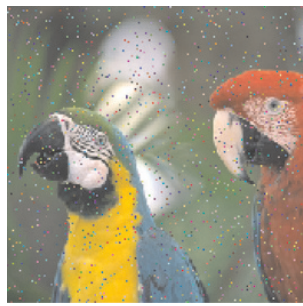

(i)

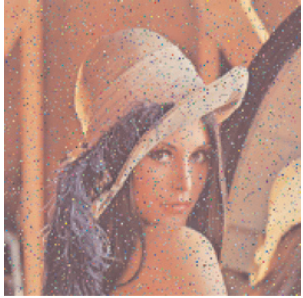

(b)

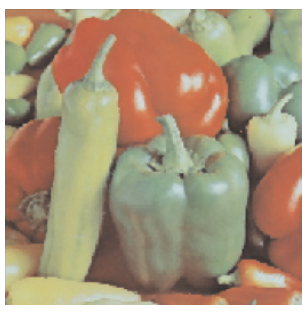

(f)

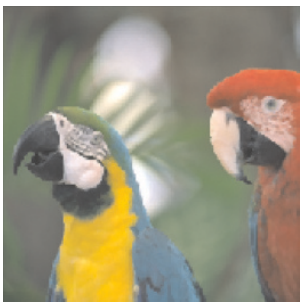

(j)

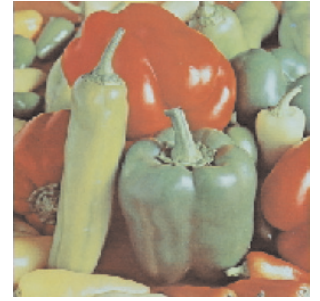

(c)

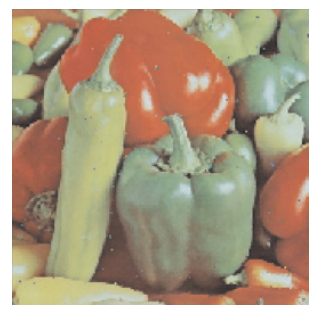

(g)

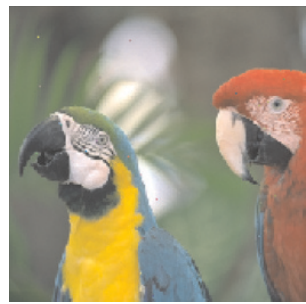

(k)

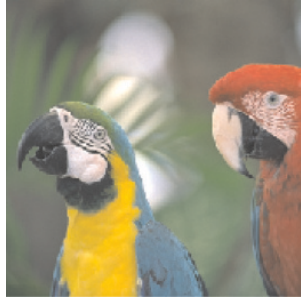

(d)

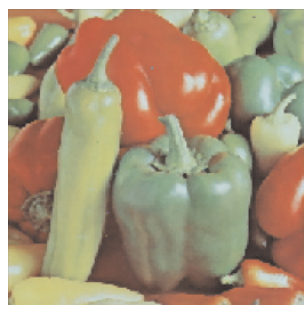

(h)

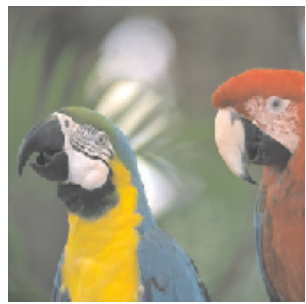

(1)

Figure 2: Color images test: (a) original image Lena, (b) image Lena corrupted by $10 \%$ impulsive noise $\left(p_{\mathbf{v}}=0.10\right)$, (c) original image Peppers, (d) original image Parrots, (e) image Peppers corrupted by $10 \%$ impulsive noise $\left(p_{\mathbf{v}}=0.10\right)$, and (i) image Parrots corrupted by $5 \%$ impulsive noise $\left(p_{\mathbf{v}}=0.05\right)$. The results obtained by filtering the images shown in Figures $2 \mathrm{e}$ and $2 \mathrm{i}$ are, respectively, (f) VMF output, (g) $\operatorname{SWVF}_{1}$ output $(p=0.5)$, (h) SWVF $\operatorname{SWtput}_{2}(p=0.5)$, (j) VMF output, (k) SWVF output $(p=0.5)$, and (l) SWVF output $_{1}(p=0)$.

where $k$, for $k=1,2, \ldots, m$, denotes the color channel, $x_{i k}$, for $i=1,2, \ldots, N$, represent the $k$ th component of the input sample $\mathbf{x}_{i}$, and med is a median operator.

Such a filter is characterized by robust noise attenuation capability and is able to remove strong image noise. Its drawback is a worse signal-detail preservation. Therefore, it can be desirable to combine noise attenuating and detail-preserving characteristics of (20) and (21). Thus, the SWVF adaptation formula is given by

$w_{i}=P\left[w_{i}+2 \mu\left(D\left(\mathbf{y}^{*}-\mathbf{y}\right)+D\left(\mathbf{x}_{(N+1) / 2}-\mathbf{y}\right)\right) \operatorname{sgn}\left(D\left(\mathbf{x}_{i}-\mathbf{y}\right)\right)\right]$.

\section{APPLICATION TO COLOR IMAGES}

In this section, the performance of the SWVFs is evaluated in the most important area of vector processing, namely, color image filtering. Note that all filtering results presented in this paper were obtained with a $3 \times 3$ square window, that is, for $N=9$. Firstly, the noise attenuation prop- erties and color/structural preservation capability of the SWVF framework are examined by utilizing the color image Lena (Figure 2a). The test image has been contaminated (Figure 2b) by impulsive noise [35] given by

$$
\mathbf{x}_{i}= \begin{cases}\mathbf{v}_{i} & \text { with probability } p_{\mathbf{v}} \\ \mathbf{o}_{i} & \text { with probability } 1-p_{\mathbf{v}}\end{cases}
$$

where $i$ characterizes the sample position, $\mathbf{o}_{i}$ is the original sample, $\mathbf{x}_{i}$ represents the sample from the noisy image and $p_{\mathbf{v}}$ is a corruption probability (also refereed as a percentage number of corrupted pixels). The impulse $\mathbf{v}_{i}=$ $\left(v_{i 1}, v_{i 2}, \ldots, v_{i m}\right)$ is independent from pixel to pixel and has generally much larger and smaller amplitude than the neighboring samples at least in one of the components.

Secondly, the SWVF filters optimized using the noisy image Lena (Figure $2 \mathrm{~b}$ ) are compared, in terms of performance related to the color images Peppers (Figure 2c) and Parrots (Figure 2d) test, with other widely used color image filters such as MF [6], VMF [15], BVDF [16], GVDF [16], SMF [16], DDF [31], and hybrid vector filters $\mathrm{HVF}_{1}, \mathrm{HVF}_{2}$ [32]. 
To provide some measure of closeness between two digital images, a number of different objective measures based on the difference in the statistical distributions of the pixel values can be utilized. In this paper, we will make the use of commonly employed objective measures [7, 19, 21] such as mean absolute error (MAE), mean square error (MSE), and normalized color difference criteria (NCD). The MAE and MSE are defined as follows:

$$
\begin{aligned}
\mathrm{MAE} & =\frac{1}{m K_{1} K_{2}} \sum_{k=1}^{m} \sum_{i=1}^{K_{1} K_{2}}\left|o_{i k}-x_{i k}\right|, \\
\mathrm{MSE} & =\frac{1}{m K_{1} K_{2}} \sum_{k=1}^{m} \sum_{i=1}^{K_{1} K_{2}}\left(o_{i k}-x_{i k}\right)^{2},
\end{aligned}
$$

where $\mathbf{o}_{i}=\left(o_{i 1}, o_{i 2}, \ldots, o_{i m}\right)$ is the original pixel, $\mathbf{x}_{i}=$ $\left(x_{i 1}, x_{i 2}, \ldots, x_{i m}\right)$ is the noisy (or restored) pixel, $i$ is the pixel position in a $K_{1} \times K_{2}$ color image, and $k$ characterizes the color channel.

To evaluate the measure of color distortion in the perceptual way to humans [2], we also use the NCD criteria defined as follows:

$$
\begin{aligned}
& \mathrm{NCD} \\
& =\frac{\sum_{i=1}^{K_{1} K_{2}} \sqrt{\left(L_{\mathbf{o}_{i}}^{*}-L_{\mathbf{x}_{i}}^{*}\right)^{2}+\left(u_{\mathbf{o}_{i}}^{*}-u_{\mathbf{x}_{i}}^{*}\right)^{2}+\left(v_{\mathbf{o}_{i}}^{*}-v_{\mathbf{x}_{i}}^{*}\right)^{2}}}{\sum_{i=1}^{K_{1} K_{2}} \sqrt{\left(L_{\mathbf{o}_{i}}^{*}\right)^{2}+\left(u_{\mathbf{o}_{i}}^{*}\right)^{2}+\left(v_{\mathbf{o}_{i}}^{*}\right)^{2}}},
\end{aligned}
$$

where $L^{*}$ represents lightness values and $\left(u^{*}, v^{*}\right)$ chrominance values corresponding to original $\mathbf{o}_{i}$ and noisy (or filtered) $\mathbf{x}_{i}$ samples expressed in the CIELUV color space.

The second evaluation approach is the subjective evaluation of the image quality with respect to the structural content (edges, textures, and fine details) preservation and the presence of unremoved impulses or introduced color artifacts as a result of faulty processing. Note that the human visual system is sensitive to changes in color, and maintaining the sharpness of the edges is as important as removing the image noise. Edges are important features since they provide an indication of the shape of the objects in the image. From this point of view, it is evident that the noise removal tasks in color images may be understood as achieving the best balance between the noise suppression and color/structural content preservation.

Tables 2 and 3 allow for the objective comparison of a variety of vector image filtering techniques. The results indicate that the proposed SWVF framework $\left(\mathrm{SWVF}_{1}\right.$ and $\mathrm{SWVF}_{2}$, both defined in (15), were trained using the test image Lena corrupted by impulsive noise $p_{\mathbf{v}}=0.10$ and 0.20 , respectively; $\mathrm{SWVF}_{3}$ is a self-adaptive technique defined by (23)) provides the best results among the tested filters, and performance of the filters designed within the SWVF framework is satisfactorily robust for a wide range of the impulsive noise corruption. Moreover, Figure 2 shows that the proposed SWVF methods achieve excellent balance between noise attenuation and signal-detail preserving characteris- tics. These results are also confirmed by the corresponding estimation errors shown in Figure 3, and zoomed parts of the test images depicted in Figures 4 and 5. It can be easily observed that the standard filtering techniques such as VMF and MFs excellently suppress impulses present in the image; however, their excessive smoothing capability results in edge blurring. In some situations, the decreased noise attenuation capability of the BVDF can result in the presence of impulses in the filtered image. It can be seen that the proposed SWVF filters achieve excellent balance between signal-detail preservation and noise suppression. This is also reflected in very small estimation error depicted in Figures $3 \mathrm{~b}, 3 \mathrm{c}, 3 \mathrm{e}$, and $3 \mathrm{f}$.

Figure 6 shows the adaptation capability of the proposed SWVF scheme (15) starting with the same initial weighting vector $\mathbf{w}=[1,1,1,1,1,1,1,1,1]$. These results are related to the training set represented by the test image Lena and its corrupted versions with $2 \%$ and $10 \%$ impulsive noise, respectively. Objective criteria MAE, MSE, and NCD are expressed in dependence on the regularization factor $\mu$ ranging from $10^{-12}$ to $10^{3}$ and the design parameter $p$ ranging from 0 to 1 . The obtained results indicate that the SWVF adaptation depends strongly on $\mu$. For its small value, the SWVF provides worse detail-preserving characteristics and performs the smoothing operation similar to the DDF, which depends on design parameter $p$. This is caused by achieved weight coefficients like in the initial weight vector $\mathbf{w}$ since too small value of $\mu$ does not have large enough influence on the adaptation of w. From Figure 7, it can be seen that the error criteria related to the SWVF performance increase with the noise corruption $p_{\mathbf{v}}$. Simultaneously, the growth of $p_{\mathbf{v}}$ results in smaller difference between the best and the worst performance of the SWVF technique related to the same noise intensity $p_{\mathbf{v}}$. To end this, Figure 8 shows the dependence of $\mu$ on $p$. It can be observed that the SWVF (for any value of $p$ ) usually achieves the best balance between all considered objective measures for $\mu$ larger than 0.5 .

Figure 9 corresponds to the convergence properties of the SWVF framework. The results show that the SWVFs converge to an appropriate weight vector within the first iteration and the additional adaptation cycle usually does not improve the performance of the SWVFs.

Figure 10 relates to the SWVF robustness. The basic SWVF ( $p=0.5$ ) was trained using the test image Lena corrupted by a wide range of impulsive noise and consecutively tested using statistically different noisy test images Peppers and Parrots. It can be seen that the training samples with low impulsive noise intensity $p_{\mathbf{v}}$ lead to worse robustness of the SWVF framework, especially in terms of the MSE values.

Apart from the numerical behavior (actual performance) of any algorithm, its computational complexity is a realistic measure of its practicality and usefulness. Therefore, the selected filtering classes are analyzed here in terms of normalized operations, such as additions (ADDs), multiplications (MULTs), divisions (DIVs), square roots (SQRTs), comparisons (COMPs), and arc cosines (ARCCOSs). Table 4 summarizes the total number of operations for VMF, BVDF, DDF, WVMF, WVDF, and SWVF schemes. Note that the SWVF is a generalized filtering class for all the 
TABLE 2: Comparison of the presented algorithms using the test image Peppers.

\begin{tabular}{|c|c|c|c|c|c|c|c|c|c|}
\hline \multirow{2}{*}{$\begin{array}{l}\text { Impulsive noise } \\
\text { Method/criterion }\end{array}$} & \multicolumn{3}{|c|}{$p_{\mathbf{v}}=0.05$} & \multicolumn{3}{|c|}{$p_{\mathbf{v}}=0.10$} & \multicolumn{3}{|c|}{$p_{\mathbf{v}}=0.15$} \\
\hline & MAE & MSE & NCD & MAE & MSE & NCD & MAE & MSE & NCD \\
\hline Noisy & 3.988 & 486.1 & 0.0441 & 7.677 & 943.3 & 0.0869 & 11.474 & 1402.4 & 0.1279 \\
\hline MF & 3.248 & 43.1 & 0.0484 & 3.579 & 53.9 & 0.0546 & 3.996 & 70.3 & 0.0620 \\
\hline VMF & 3.169 & 43.9 & 0.0452 & 3.503 & 55.0 & 0.0494 & 3.858 & 68.7 & 0.0540 \\
\hline BVDF & 3.740 & 60.7 & 0.0438 & 4.151 & 82.7 & 0.0484 & 4.598 & 113.2 & 0.0532 \\
\hline DDF & 3.182 & 44.6 & 0.0431 & 3.512 & 56.6 & 0.0475 & 3.844 & 70.8 & 0.0518 \\
\hline GVDF & 3.433 & 57.9 & 0.0453 & 3.785 & 73.4 & 0.0492 & 4.139 & 92.0 & 0.0534 \\
\hline SMF & 3.442 & 42.9 & 0.0456 & 3.723 & 52.3 & 0.0499 & 4.101 & 64.8 & 0.0545 \\
\hline $\mathrm{HVF}_{1}$ & 3.282 & 42.9 & 0.0441 & 3.626 & 53.6 & 0.0485 & 3.992 & 68.0 & 0.0530 \\
\hline $\mathrm{HVF}_{2}$ & 3.274 & 41.9 & 0.0441 & 3.614 & 52.4 & 0.0485 & 3.994 & 67.2 & 0.0530 \\
\hline $\operatorname{SWVF}_{1}(p=0)$ & 0.995 & 19.9 & 0.0138 & 1.460 & 50.7 & 0.0203 & 2.221 & 114.1 & 0.0299 \\
\hline $\operatorname{SWVF}_{1}(p=0.5)$ & 0.962 & 18.1 & 0.142 & 1.381 & 43.1 & 0.0196 & 2.088 & 88.4 & 0.0275 \\
\hline $\operatorname{SWVF}_{1}(p=1)$ & 1.595 & 31.0 & 0.0193 & 2.068 & 65.5 & 0.0244 & 2.667 & 113.0 & 0.0311 \\
\hline $\operatorname{SWVF}_{2}(p=0)$ & 1.454 & 21.2 & 0.0204 & 1.754 & 33.3 & 0.250 & 2.189 & 56.1 & 0.0309 \\
\hline $\operatorname{SWVF}_{2}(p=0.5)$ & 1.783 & 24.0 & 0.0255 & 2.068 & 35.1 & 0.0295 & 2.448 & 55.1 & 0.0346 \\
\hline $\operatorname{SWVF}_{2}(p=1)$ & 2.522 & 39.3 & 0.0295 & 2.879 & 58.5 & 0.0338 & 3.346 & 93.3 & 0.0390 \\
\hline $\operatorname{SWVF}_{3}(p=0)$ & 2.032 & 27.7 & 0.0287 & 2.409 & 37.4 & 0.0345 & 2.916 & 52.9 & 0.0414 \\
\hline $\operatorname{SWVF}_{3}(p=0.5)$ & 2.378 & 33.0 & 0.0329 & 2.734 & 42.6 & 0.0378 & 3.201 & 58.5 & 0.0436 \\
\hline $\operatorname{SWVF}_{3}(p=1)$ & 3.241 & 53.4 & 0.0377 & 3.894 & 79.4 & 0.0456 & 4.170 & 100.4 & 0.0484 \\
\hline
\end{tabular}

TABLe 3: Comparison of the presented algorithms using the test image Parrots.

\begin{tabular}{|c|c|c|c|c|c|c|c|c|c|}
\hline \multirow{2}{*}{$\begin{array}{l}\text { Impulsive noise } \\
\text { Method/criterion }\end{array}$} & \multicolumn{3}{|c|}{$p_{\mathbf{v}}=0.05$} & \multicolumn{3}{|c|}{$p_{\mathbf{v}}=0.10$} & \multicolumn{3}{|c|}{$p_{\mathbf{v}}=0.15$} \\
\hline & MAE & MSE & NCD & MAE & MSE & NCD & MAE & MSE & NCD \\
\hline Noisy & 3.805 & 443.6 & 0.0432 & 7.526 & 882.0 & 0.0857 & 11.115 & 1311.5 & 0.1270 \\
\hline MF & 2.718 & 63.1 & 0.0170 & 2.960 & 70.0 & 0.0198 & 3.275 & 80.9 & 0.0236 \\
\hline VMF & 2.669 & 64.2 & 0.0132 & 2.890 & 69.6 & 0.0142 & 3.178 & 80.0 & 0.0158 \\
\hline BVDF & 3.460 & 109.0 & 0.0116 & 3.630 & 113.5 & 0.0127 & 3.883 & 125.2 & 0.0144 \\
\hline DDF & 2.645 & 65.3 & 0.0117 & 2.839 & 69.7 & 0.0128 & 3.070 & 76.7 & 0.0143 \\
\hline GVDF & 3.036 & 93.6 & 0.0126 & 3.188 & 96.2 & 0.0137 & 3.321 & 97.0 & 0.0165 \\
\hline SMF & 2.927 & 61.6 & 0.0130 & 3.133 & 67.1 & 0.0141 & 3.492 & 87.5 & 0.0153 \\
\hline $\mathrm{HVF}_{1}$ & 2.786 & 65.7 & 0.0122 & 3.002 & 69.9 & 0.0132 & 2.786 & 65.7 & 0.0122 \\
\hline $\mathrm{HVF}_{2}$ & 2.771 & 63.5 & 0.0121 & 2.999 & 68.6 & 0.0131 & 2.771 & 63.5 & 0.0121 \\
\hline $\operatorname{SWVF}_{1}(p=0)$ & 0.903 & 27.1 & 0.0042 & 1.267 & 47.2 & 0.0067 & 1.942 & 104.1 & 0.0129 \\
\hline $\operatorname{SWVF}_{1}(p=0.5)$ & 0.745 & 18.5 & 0.0033 & 1.021 & 29.8 & 0.0049 & 1.539 & 67.2 & 0.0086 \\
\hline $\operatorname{SWVF}_{1}(p=1)$ & 1.373 & 43.2 & 0.0046 & 1.611 & 53.6 & 0.0058 & 2.065 & 88.9 & 0.0087 \\
\hline $\operatorname{SWVF}_{2}(p=0)$ & 1.256 & 30.9 & 0.0056 & 1.501 & 41.6 & 0.0069 & 1.857 & 60.9 & 0.0095 \\
\hline $\operatorname{SWVF}_{2}(p=0.5)$ & 1.399 & 36.0 & 0.0058 & 1.624 & 43.4 & 0.0070 & 1.881 & 53.3 & 0.0086 \\
\hline $\operatorname{SWVF}_{2}(p=1)$ & 2.199 & 70.0 & 0.0070 & 2.385 & 75.2 & 0.0081 & 2.643 & 89.5 & 0.0099 \\
\hline $\operatorname{SWVF}_{3}(p=0)$ & 1.581 & 38.1 & 0.0072 & 1.909 & 46.7 & 0.0090 & 2.229 & 56.4 & 0.0110 \\
\hline $\mathrm{SWVF}_{3}(p=0.5)$ & 2.098 & 51.8 & 0.0091 & 2.370 & 58.2 & 0.0105 & 2.691 & 67.1 & 0.0124 \\
\hline $\operatorname{SWVF}_{3}(p=1)$ & 3.380 & 105.2 & 0.0113 & 3.559 & 111.2 & 0.0124 & 3.748 & 120.4 & 0.0139 \\
\hline
\end{tabular}

above-mentioned filtering classes and thus, it is expected that the proposed generalization will increase the overall cost. As it can be seen, the VMF is a computationally most attractive case among the considered filtering schemes.
Summarizing the results presented above, the following conclusions can be drawn: (i) the proposed SWVF optimization framework holds excellent adaptation capability; (ii) the noise attenuation capability of the SWVF filters 


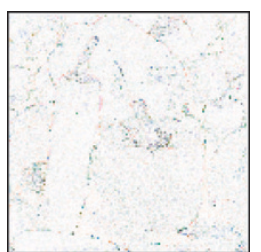

(a)

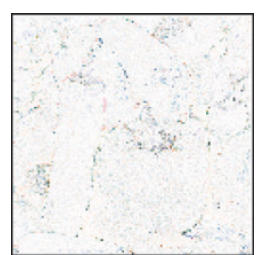

(b)

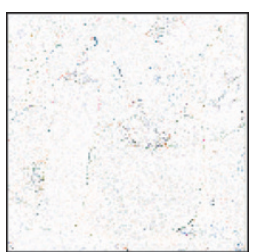

(c)

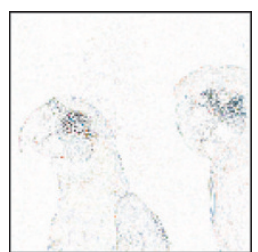

(d)

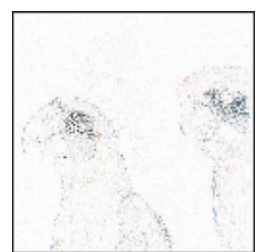

(e)

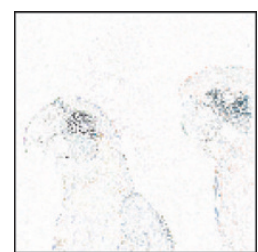

(f)

FIGURE 3: Estimation error corresponding to filtering of the images shown in Figures 2e and 2i, respectively: ((a), (d)) VMF output; ((b), (e)) $\mathrm{SWVF}_{1}$ output $(p=0.5)$; ((c), (f)) $\mathrm{SWVF}_{3}$ output $(p=0)$.

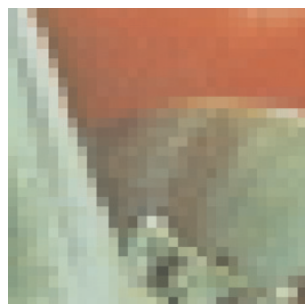

(a)

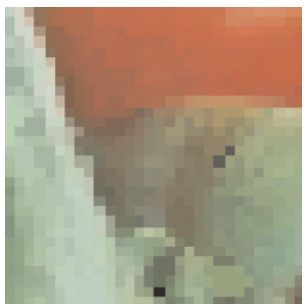

(e)

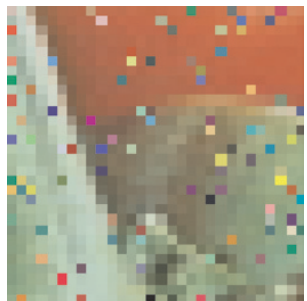

(b)

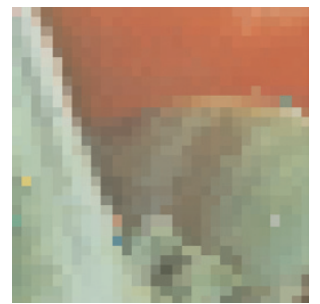

(f)

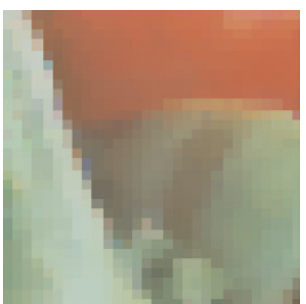

(c)

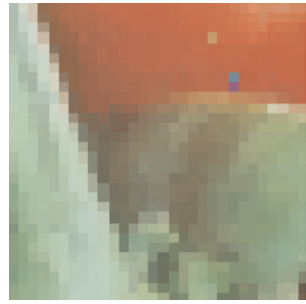

(g)

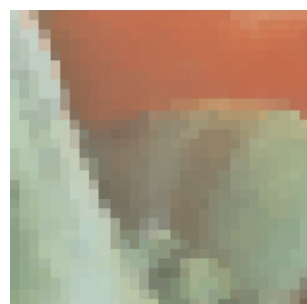

(d)

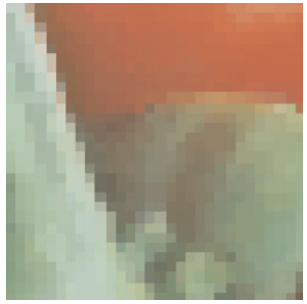

(h)

FIGURE 4: Zoomed parts of images corresponding to filtering of Figure 2e: (a) original image, (b) noisy image $\left(p_{\mathbf{v}}=0.10\right),(\mathrm{c})$ MF output, (d) VMF output, (e) BVDF output, (f) $\operatorname{SWVF}_{1}$ output $(p=0.5)$, (g) SWVF output $(p=0.5)$, and (h) SWVF $\operatorname{SWutput}_{3}(p=0)$.

increases with the degree of the impulsive noise corruption present in the training set; (iii) the proposed SWVFs attenuate impulsive noise present in the color images while excellently preserving color/structural information; (iv) SWVF filters are sufficiently robust and provide better results than widely used image filtering schemes, both vectorial in nature and componentwise; (v) the filter complexity is comparable with widely used vectorial schemes; (vi) the proposed multichannel adaptation tool is relatively fast, for example, in the case of a $256 \times 256$ color image less than 1 second using Intel PIV $2.4 \mathrm{GHz}$ processor and program written in $\mathrm{C}$ on the MS VC++ 5.0 platform, and is easy to implement.

\section{CONCLUSION}

The paper introduced a generalized vector signal processing framework based on the order-statistics theory. The proposed SWVF framework generalizes many attractive vector processing schemes by selecting filtering parameters in a sim- ple, consistent, and systematic manner. The behavior of the introduced vector processor was analyzed in details. Color image filtering was introduced as an example of important vectorial processing. In order to adapt the filter characteristics to varied signal and noise statistics, a new generalized multichannel weight adaptation was introduced. This adaptation tool based on the sigmoidal function is fast, easy to implement, and achieves convergence within the first few iterations. Simulation results and comparisons reported here indicate that the proposed class is able to achieve excellent tradeoff between smoothing and detail preservation, is sufficiently robust, and it can outperform the commonly used filtering schemes in terms of both objective and subjective evaluation approaches.

Future research will focus on the introduction of alternative weight adaptation formulas and mechanisms. It is expected that this modification in conjunction with a separate adaptation of the filtering weights related to magnitude and angular characteristics of the vectorial inputs will enhance further the performance and provide additional flexibility. 


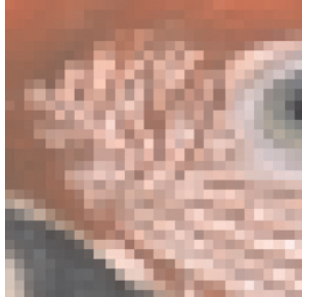

(a)

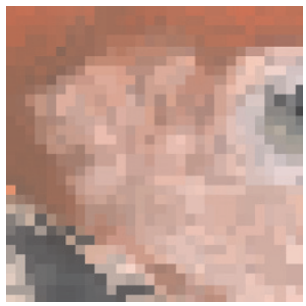

(e)

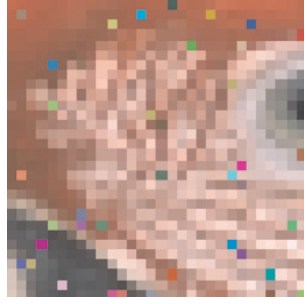

(b)

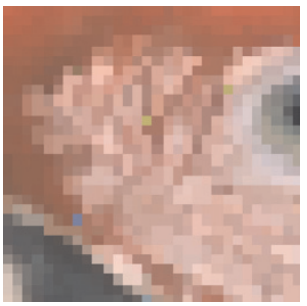

(f)

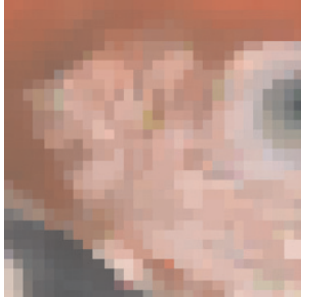

(c)

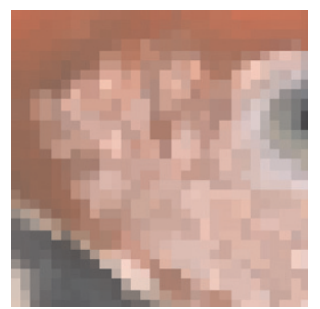

(g)

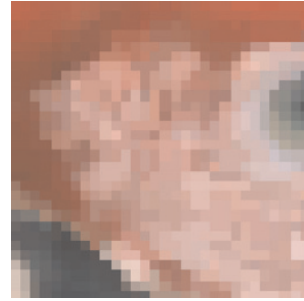

(d)

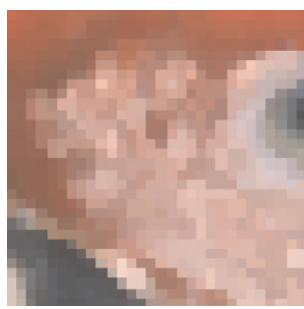

(h)

FIGURE 5: Zoomed parts of images corresponding to filtering of Figure 2i: (a) original image, (b) noisy image $\left(p_{\mathbf{v}}=0.05\right)$, (c) MF output, (d) VMF output, (e) BVDF output, (f) $\operatorname{SWVF}_{1}$ output $(p=0.5)$, (g) $\operatorname{SWVF}_{2}$ output $(p=0.5)$, and (h) SWVF $\mathrm{Sutput}_{3}(p=0)$.

\section{APPENDIX}

Let the weight vector $\mathbf{w}=\left\{w_{1}, w_{2}, \ldots, w_{N}\right\}$ define the componentwise WM filter which is separately applied to the RGB color channels. In this case, $W_{k}=\left\{x_{1 k}, x_{2 k}, \ldots, x_{N k}\right\}$ denotes the input set, while $y_{k}=y\left(\mathbf{w}, W_{k}\right)$ is the WM output for the $k$ th color channel. Given an estimation error $e_{k}=o_{k}-y_{k}$, the cost function defined under the MAE is expressed as $J_{\text {MAE }}(\mathbf{w})=E\left\{\left|o_{k}-y\left(\mathbf{w}, W_{k}\right)\right|\right\}$, where $E\{\cdot\}$ represents statistical expectation. Similarly to (14), the constrained solution [18] leads to the minimization of $J_{\mathrm{MAE}}(\mathbf{w})$ with simultaneous consideration of $w_{i} \geq 0$, for $i=1,2, \ldots, N$.

Assuming that $J_{\mathrm{MAE}}(\mathbf{w})$ is nonconvex in the weights and the optimal weights are at one of the multiple local minima, the conditions for optimality can be derived as follows:

$$
\frac{\partial J_{\mathrm{MAE}}(\mathbf{w})}{\partial w_{i}}=E\left\{\operatorname{sgn}\left(o_{k}-y_{k}\right) \frac{\partial y_{k}}{\partial w_{i}}\right\}
$$

where $\operatorname{sgn}\left(o_{k}-y_{k}\right)$ is the sign function equal to 1,0 , and -1 for $\left(o_{k}-y_{k}\right)>0,\left(o_{k}-y_{k}\right)=0$, and $\left(o_{k}-y_{k}\right)<0$, respectively.

Based on the WM optimization framework [18], minimization of (A.1) leads to the adaptation step defined as follows:

$$
w_{i}=P\left[w_{i}+2 \mu \frac{\partial J(\mathbf{w})}{\partial w_{i}}\right]
$$

where $i=1,2, \ldots, N$, and $P(\cdot)$ is defined in (17). Replacing the expectation operator with the instantaneous estimates results in the following adaptation formula:

$$
w_{i}=P\left[w_{i}+2 \mu \frac{\partial y(\mathbf{w})}{\partial w_{i}} \operatorname{sgn}\left(o_{k}-y_{k}\right)\right]
$$

where $e_{k}=o_{k}-y_{k}$ denotes the error of the actual output. Assuming the sigmoidal approximation of the sign function [18], another simplification of (A.3) results in (18).

To extend (18) to vector arrays such as the color images, the differences between the $k$ th components should be replaced with the distances between the vector-valued inputs. There exist at least two ways to obtain the vector extension of (18). By modifying (18) as follows:

$$
w_{i}=P\left[w_{i}-2 \mu\left(y_{k}-o_{k}\right) \operatorname{sgn}\left(\left(x_{i k}-o_{k}\right)-\left(y_{k}-o_{k}\right)\right)\right],
$$

the first solution reduces to

$$
w_{i}=P\left[w_{i}-2 \mu \psi(\mathbf{y}, \mathbf{o}) \operatorname{sgn}\left(\psi\left(\mathbf{x}_{i}, \mathbf{o}\right)-\psi(\mathbf{y}, \mathbf{o})\right)\right],
$$

where $\psi(\mathbf{a}, \mathbf{b})=\left(\|\mathbf{a}-\mathbf{b}\|_{L}\right)^{1-p}(A(\mathbf{a}, \mathbf{b}))^{p}$ denotes the error criterion corresponding to the proposed SWVF scheme. It can be emphasized that (A.5) is numerically stable, and depending on $\mu$ and $p$, the obtained SWVF weight vector $\mathbf{w}$ can represent the robust solution.

However, assuming $\operatorname{sgn}(\cdot)$ of (A.5) is equal to 1 for a moment, (A.5) reduces to $w_{i}=P\left[w_{i}-2 \mu \psi(\mathbf{y}, \mathbf{o})\right]$. Since this development ignores the sign of the difference, which is essential for calculation of the weights as shown in (19), the approach of (A.5) often results in zero (or very small) weighting coefficients. Therefore, the solution used throughout the paper utilizes the sign error criterion between the vectorial inputs $\mathbf{a}$ and $\mathbf{b}$. Based on $\psi(\mathbf{a}, \mathbf{b})$ and the polarity function $S(\mathbf{a}, \mathbf{b})$ of $(11)$, the error criterion corresponding to the SWVF framework is expressed as $D(\mathbf{a}-\mathbf{b})=S(\mathbf{a}, \mathbf{b}) \psi(\mathbf{a}, \mathbf{b})$, resulting in (10). Using $D(\mathbf{a}-\mathbf{b})$ as the error function, the scalar optimization formula (18) can be extended for the vector-valued case (15) utilized in this paper. 


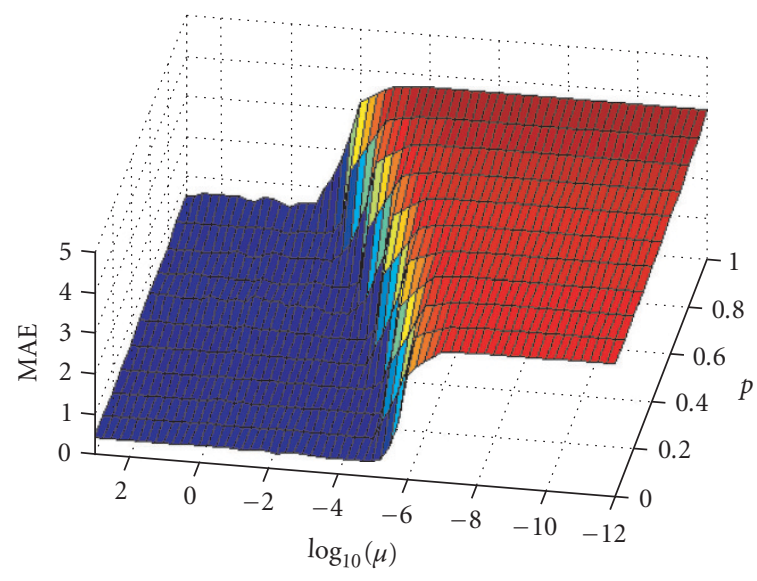

(a)

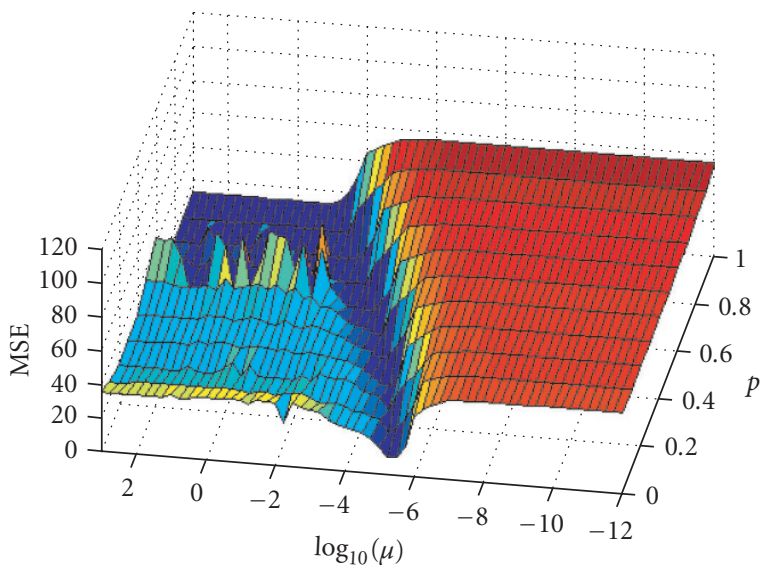

(c)

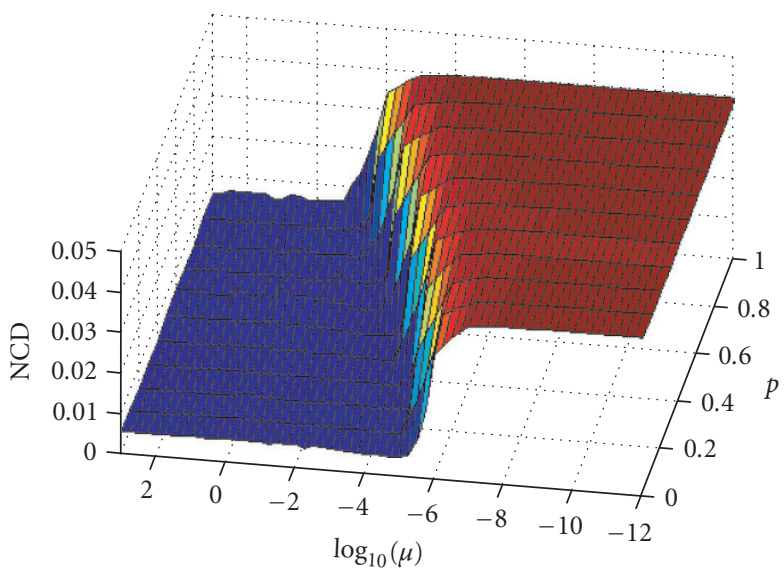

(e)



(b)

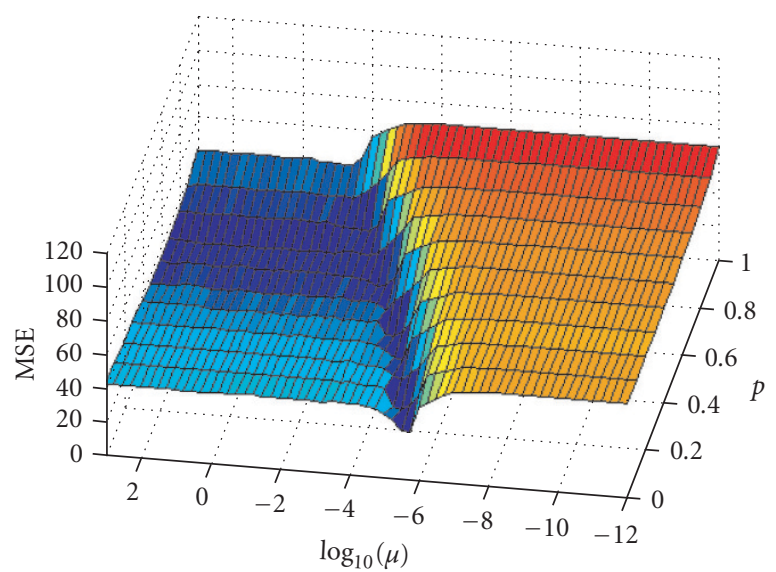

(d)

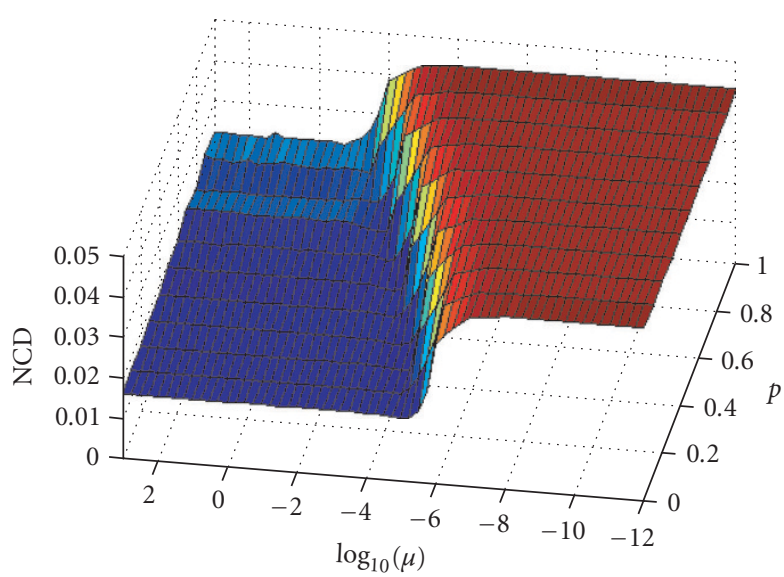

(f)

Figure 6: SWVF weight adaptation expressed through ((a), (b)) the MAE, ((c), (d)) the MSE, and ((e), (f)) the NCD image quality measures in the dependence on the regularization factor $\mu$ and design parameter $p$. Training set was obtained through the image Lena with ((a), (c), (e) $2 \%$ impulsive noise $\left(p_{\mathbf{v}}=0.02\right)$; ((b), (d), (f)) $10 \%$ impulsive noise $\left(p_{\mathbf{v}}=0.10\right)$. 


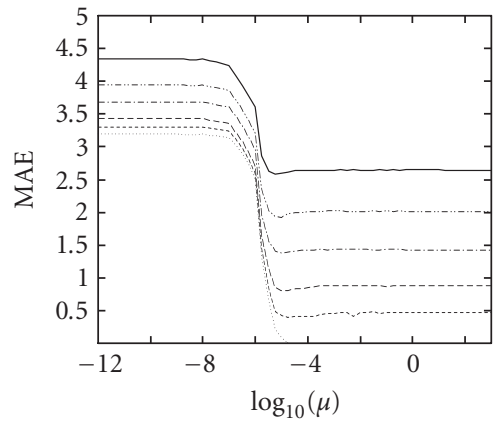

(a)

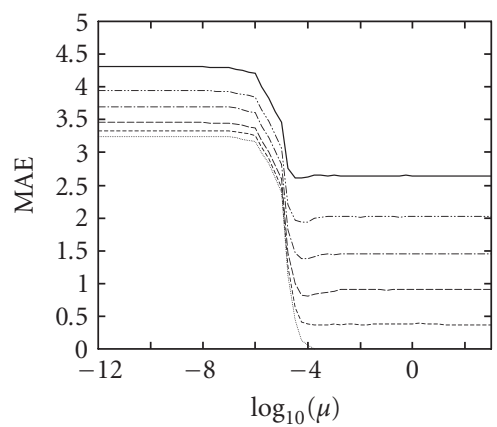

(b)

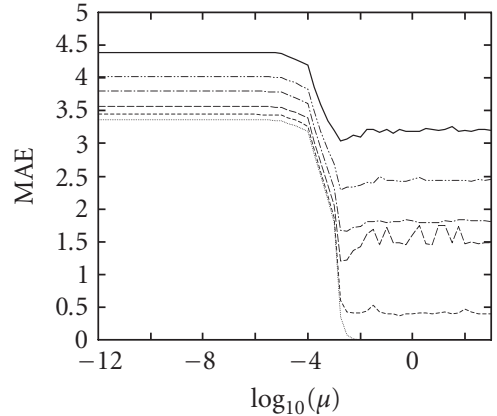

(c)

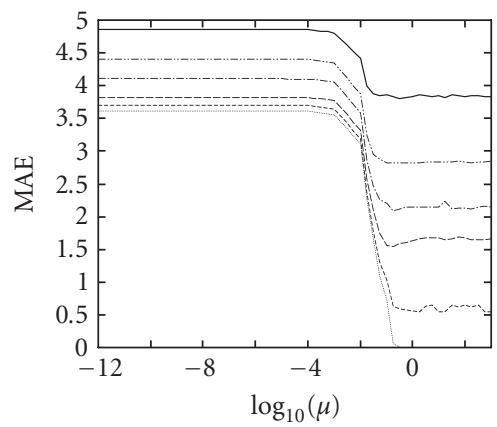

(d)

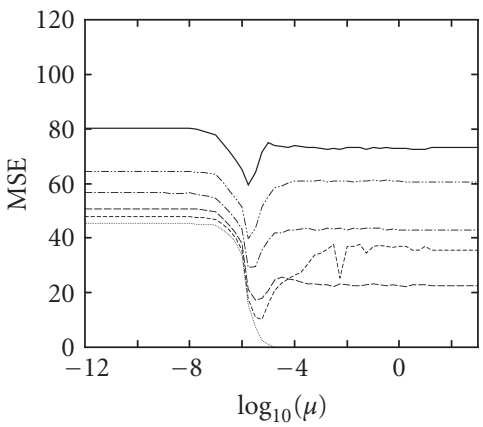

(e)

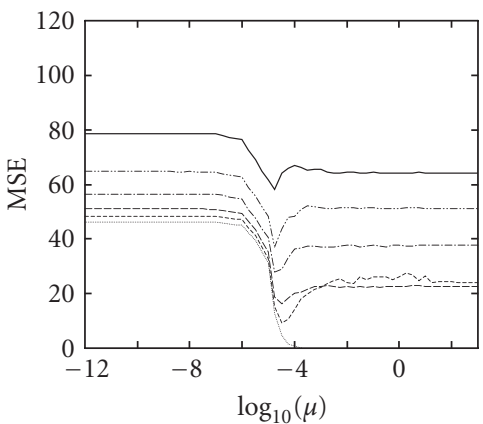

(f)

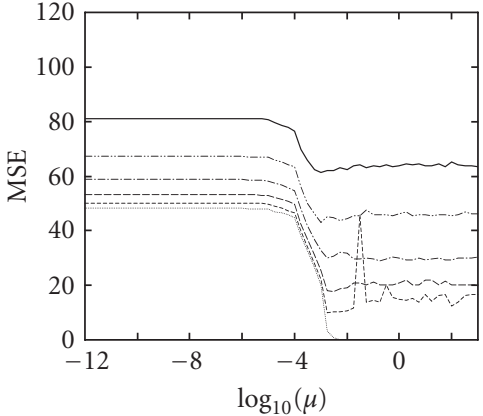

(g)

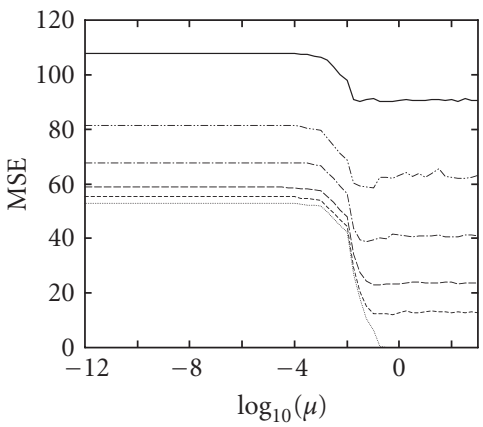

(h)

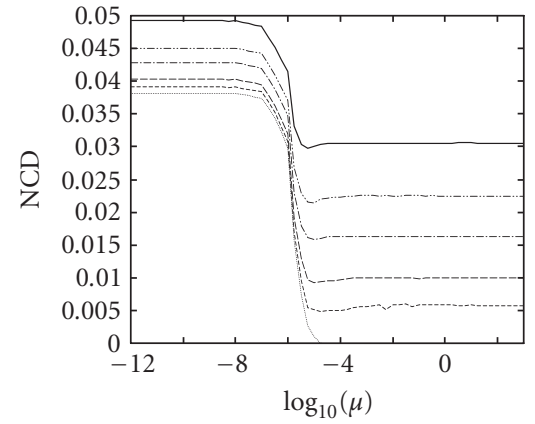

(i)

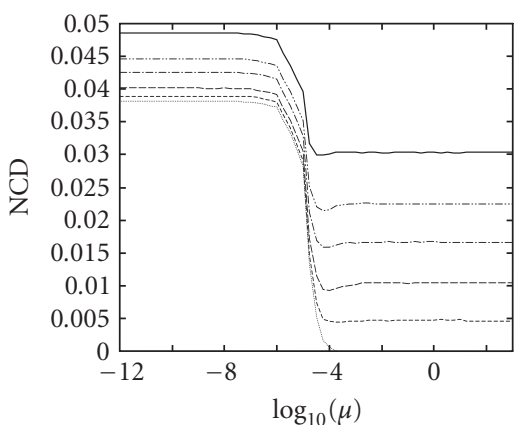

(j)

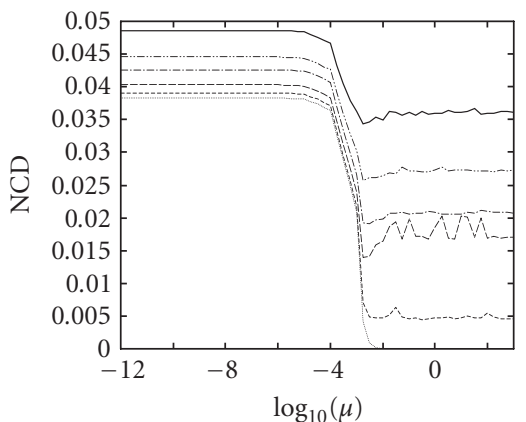

(k)

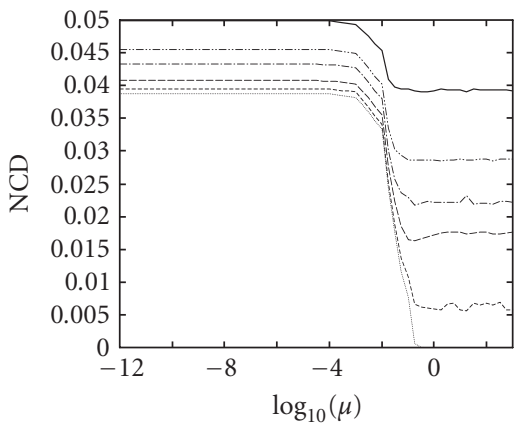

(l)

$$
\begin{aligned}
& \longrightarrow p_{v}=0 \\
& \text {---- } p_{v}=0.05 \\
& \text {-.-.. } p_{v}=0.10 \\
& \begin{aligned}
-\cdots-p_{v} & =0.15 \\
-p_{v} & =0.20
\end{aligned}
\end{aligned}
$$

FIGURE 7: Comparison of the SWVF adaptation capability expressed via ((a)-(d)) MAE, ((e)-(h)) MSE, and ((i)-(l)) NCD error criteria using the different noise probability $p_{\mathrm{v}}$ in the dependence on the regularization factor $\mu$ : ((a), (e), (i)) $p=0$; ((b), (f), (j)) $p=0.3$; ((c), (g), (k)) $p=0.7$; and ((d), (h), (l)) $p=1$. 


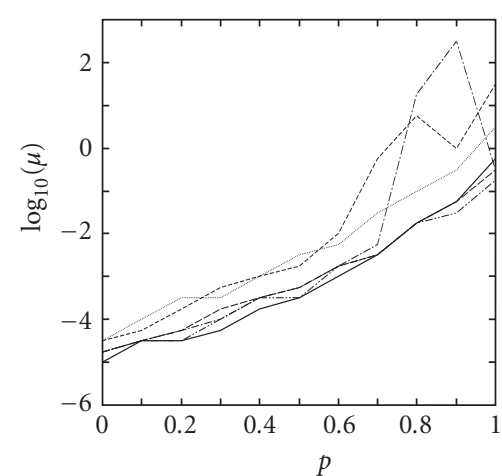

(a)

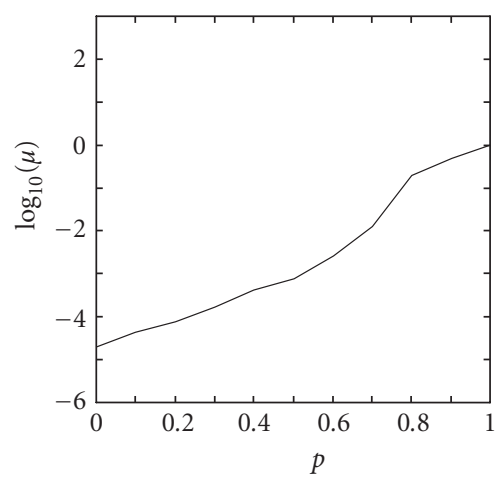

(d)

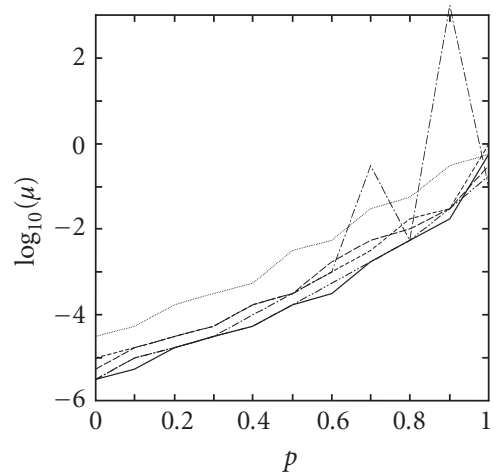

(b)

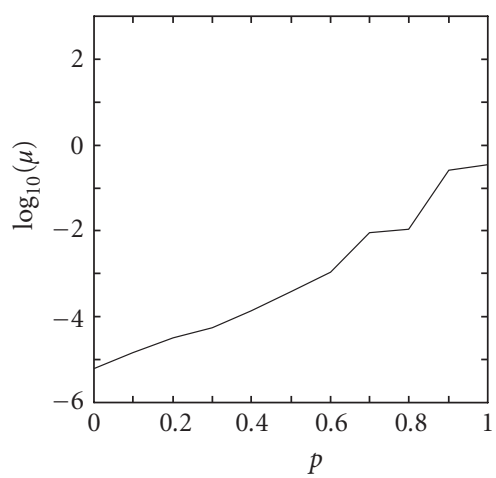

(e)

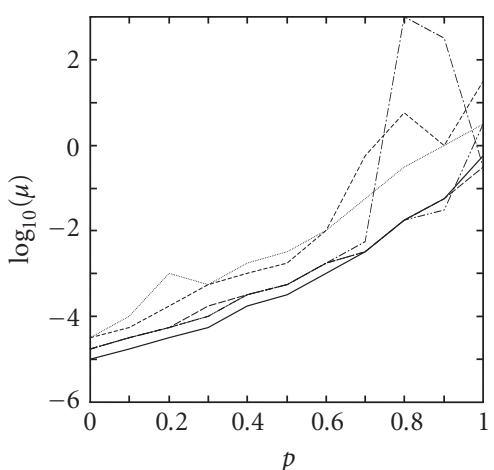

(c)

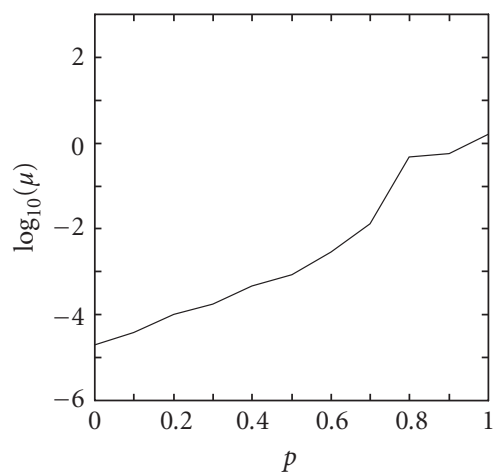

(f)

$$
\begin{aligned}
& \begin{aligned}
p_{v} & =0 \\
\cdots & p_{v}=0.02
\end{aligned} \\
& \text {---- } p_{v}=0.05 \\
& \text {-.... } p_{v}=0.10 \\
& \begin{aligned}
-\cdots-p_{v} & =0.15 \\
-p_{v} & =0.20
\end{aligned}
\end{aligned}
$$

Figure 8: Optimal values of regularization factor $\mu$ versus design parameter $p$ : ((a), (c), (e)) achieved values related to objective measures (a) MAE, (c) MSE, and (e) NCD using different noise intensity $p_{\mathbf{v}}$; ((b), (d), (f)) approximated suboptimal values for a wide range of $p_{\mathbf{v}}$.

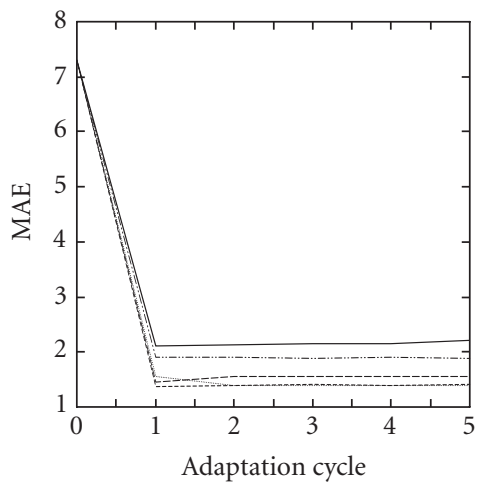

(a)

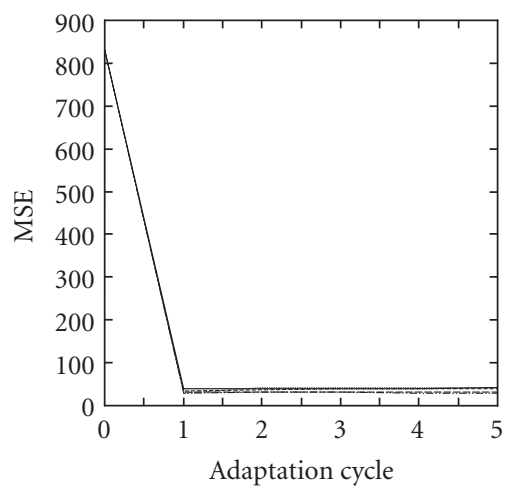

(b)

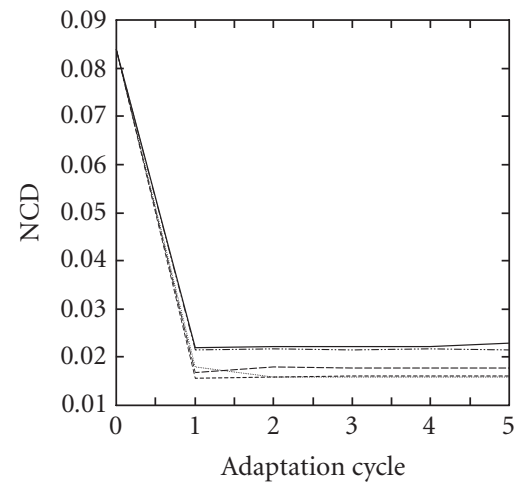

(c)

$$
\begin{aligned}
& p=0 \\
& \text { …- } p=0.25 \\
& \begin{array}{l}
--p=0.5 \\
-\cdots p=0.75
\end{array}
\end{aligned}
$$$$
-p=1
$$

FIGURE 9: Adaptation capability of the proposed optimization framework (demonstrated using the image Lena corrupted by $10 \%$ impulsive noise) depending on the number of iterations: (a) MAE, (b) MSE, and (c) NCD. 


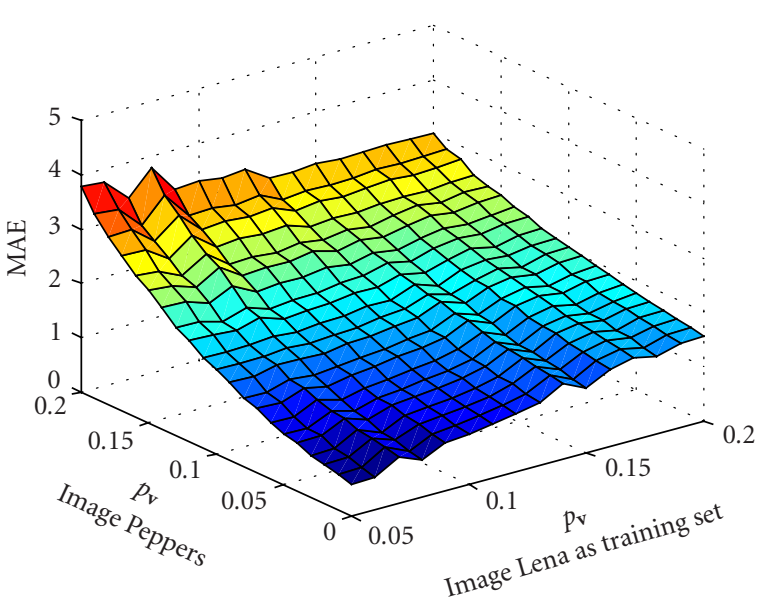

(a)

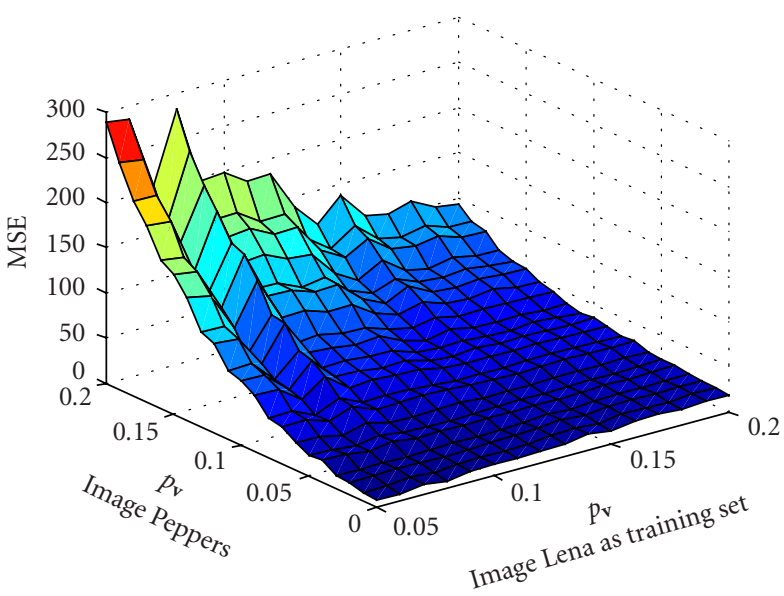

(c)

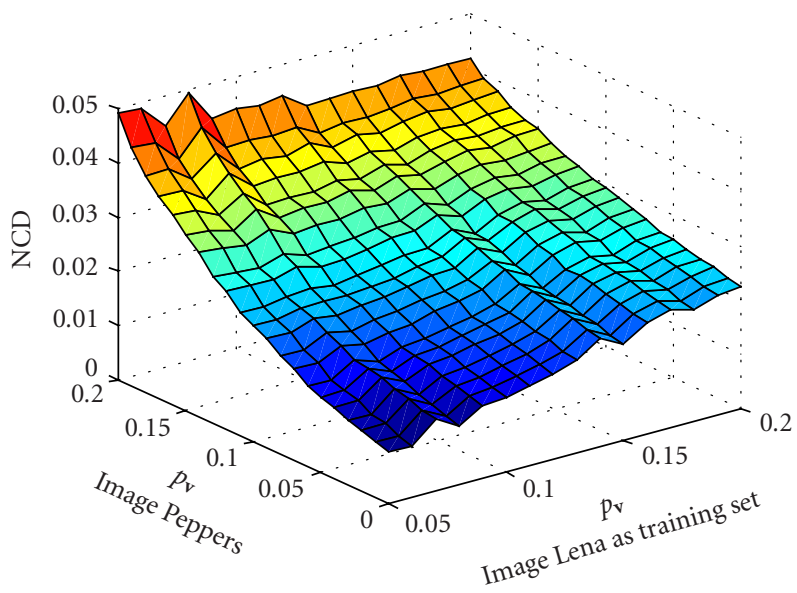

(e)

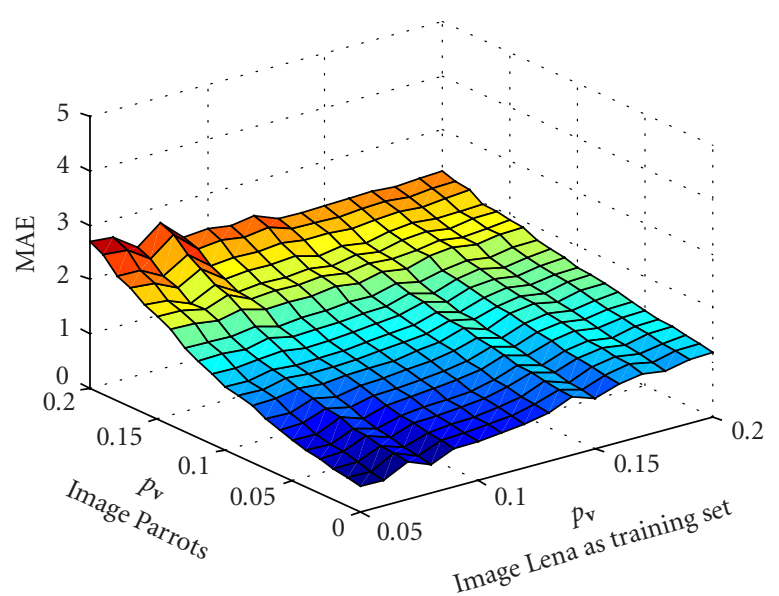

(b)

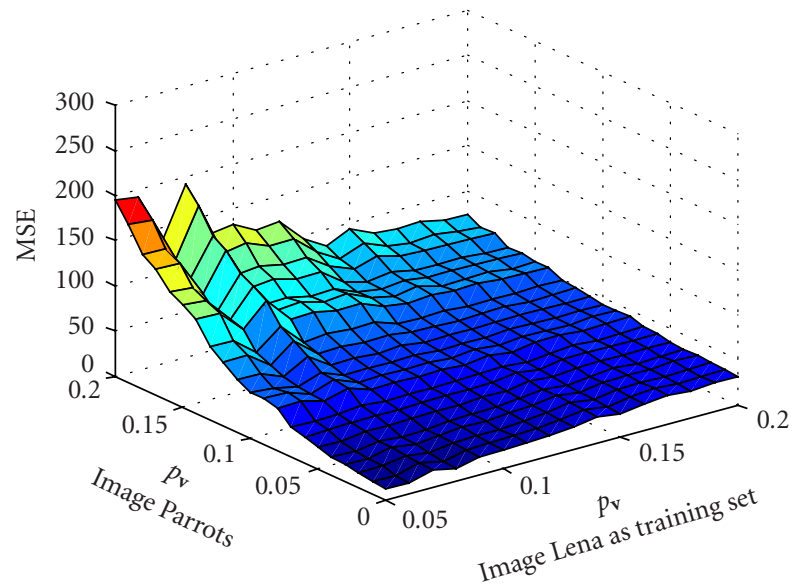

(d)

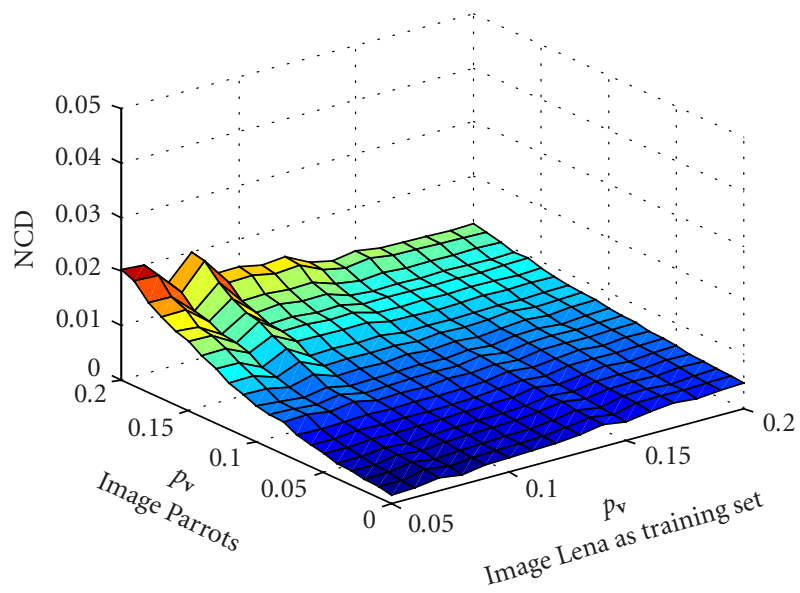

(f)

Figure 10: Performance of the SWVF technique (for $p=0.5)$ related to the test images Peppers ((a), (c), (e)) and Parrots ((b), (d), (f)) corrupted by a wide range of impulsive noise $p_{\mathbf{v}}$ in dependence on the impulsive noise corruption $p_{\mathbf{v}}$ affecting the training image Lena. 
TABLE 4: Number of elementary operations for a complete processing cycle corresponding to a $3 \times 3$ supporting window.

\begin{tabular}{|c|c|c|c|c|c|c|}
\hline Filter/operation & ADDs & MULTs & DIVs & SQRTs & COMPs & ARCCOSs \\
\hline VMF & 186 & 63 & - & 21 & 8 & - \\
\hline BVDF & 375 & 210 & 21 & 21 & 8 & 21 \\
\hline DDF & 540 & 282 & 21 & 42 & 8 & 21 \\
\hline WVMF & 186 & 135 & - & 21 & 8 & - \\
\hline WVDF & 375 & 282 & 21 & 21 & 8 & 21 \\
\hline SWVF & 540 & 426 & 21 & 42 & 8 & 21 \\
\hline
\end{tabular}

\section{ACKNOWLEDGEMENT}

The work of the first author is supported by a NATO/NSERC Science Award.

\section{REFERENCES}

[1] R. Lukac, B. Smolka, K. Martin, K. N. Plataniotis, and A. N. Venetsanopoulos, "Vector filtering for color imaging," to appear in IEEE Signal Processing Magazine, Special issue on color image processing.

[2] K. N. Plataniotis and A. N. Venetsanopoulos, Color Image Processing and Applications, Springer-Verlag, Berlin, Germany, 2000.

[3] J. Li, K. Yu, T. He, Y. Lin, S. Li, and Y.-Q. Zhang, "Scalable portrait video for mobile video communication," IEEE Trans. Circuits and Systems for Video Technology, vol. 13, no. 5, pp. 376-384, 2003.

[4] O. Faugeras, "Digital color image processing within the framework of a human visual model," IEEE Trans. Acoustics, Speech, and Signal Processing, vol. 27, no. 4, pp. 380-393, 1979.

[5] H. Rantanen, M. Karlsson, P. Pohjala, and S. Kalli, "Color video signal processing with median filters," IEEE Transactions on Consumer Electronics, vol. 38, no. 3, pp. 157-161, 1992.

[6] J. Zheng, K. P. Valavanis, and J. M. Gauch, "Noise removal from color images," Journal of Intelligent and Robotic Systems, vol. 7, no. 3, pp. 257-285, 1993.

[7] B. Smolka, K. N. Plataniotis, and A. N. Venetsanopoulos, "Nonlinear techniques for color image processing," in Nonlinear Signal and Image Processing: Theory, Methods, and Applications, K. E. Barner and G. R. Arce, Eds., pp. 445-505, CRC Press, Boca Raton, Fla, USA, 2004.

[8] J. Astola and P. Kuosmanen, Fundamentals of Nonlinear Digital Filtering, CRC Press, Roca Baton, Fla, USA, 1997.

[9] S. K. Mitra and G. L. Sicuranza, Nonlinear Image Processing, Academic Press, London, UK, 2001.

[10] I. Pitas and A. N. Venetsanopoulos, Nonlinear Digital Filters: Principles and Applications, Kluwer Academic Publishers, Boston, Mass, USA, 1990.

[11] I. Pitas and A. N. Venetsanopoulos, "Order statistics in digital image processing," Proceedings of the IEEE, vol. 80, no. 12, pp. 1893-1921, 1992.

[12] R. C. Hardie and G. R. Arce, "Ranking in $R^{p}$ and its use in multivariate image estimation," IEEE Trans. Circuits and Systems for Video Technology, vol. 1, no. 2, pp. 197-209, 1991.

[13] B. Smolka, R. Lukac, A. Chydzinski, K. N. Plataniotis, and W. Wojciechowski, "Fast adaptive similarity based impulsive noise reduction filter," Real-Time Imaging, vol. 9, no. 4, pp. 261-276, 2003.
[14] K. Tang, J. Astola, and Y. Neuvo, "Nonlinear multivariate image filtering techniques," IEEE Trans. Image Processing, vol. 4, no. 6, pp. 788-798, 1995.

[15] J. Astola, P. Haavisto, and Y. Neuvo, "Vector median filters," Proceedings of the IEEE, vol. 78, no. 4, pp. 678-689, 1990.

[16] P. E. Trahanias, D. Karakos, and A. N. Venetsanopoulos, "Directional processing of color images: theory and experimental results," IEEE Trans. Image Processing, vol. 5, no. 6, pp. 868$880,1996$.

[17] R. Lukac, K. N. Plataniotis, B. Smolka, and A. N. Venetsanopoulos, "Generalized sigmoidal optimization of selection weighted vector filters," in Proc. IEEE-EURASIP Workshop on Nonlinear Signal and Image Processing (NSIP '03), Grado, Italy, June 2003.

[18] L. Yin and Y. Neuvo, "Fast adaptation and performance characteristics of FIR-WOS hybrid filters," IEEE Trans. Image Processing, vol. 42, no. 7, pp. 1610-1628, 1994.

[19] K. N. Plataniotis, D. Androutsos, and A. N. Venetsanopoulos, "Adaptive fuzzy systems for multichannel signal processing," Proceedings of the IEEE, vol. 87, no. 9, pp. 1601-1622, 1999.

[20] R. O. Duda, P. E. Hart, and D. G. Stork, Pattern Classification and Scene Analysis, John Wiley \& Sons, New York, NY, USA, 2nd edition, 2000.

[21] R. Lukac, "Adaptive vector median filtering," Pattern Recognition Letters, vol. 24, no. 12, pp. 1889-1899, 2003.

[22] L. Lucat, P. Siohan, and D. Barba, "Adaptive and global optimization methods for weighted vector median filters," Signal Processing: Image Communication, vol. 17, no. 7, pp. 509-524, 2002.

[23] T. Viero, K. Oistamo, and Y. Neuvo, "Three-dimensional median-related filters for color image sequence filtering," IEEE Trans. Circuits and Systems for Video Technology, vol. 4, no. 2, pp. 129-142, 208-210, 1994.

[24] R.-S. Lin and Y.-C. Hsueh, "Multichannel filtering by gradient information," Signal Processing, vol. 80, no. 2, pp. 279-293, 2000.

[25] L. Khriji and M. Gabbouj, "Vector median-rational hybrid filters for multichannel image processing," IEEE Signal Processing Letters, vol. 6, no. 7, pp. 186-190, 1999.

[26] L. Khriji and M. Gabbouj, "Adaptive fuzzy order statisticsrational hybrid filters for color image processing," Fuzzy Sets and Systems, vol. 128, no. 1, pp. 35-46, 2002.

[27] H. H. Tsai and P. T. Yu, "Genetic-based fuzzy hybrid multichannel filters for color image restoration," Fuzzy Sets and Systems, vol. 114, no. 2, pp. 203-224, 2000.

[28] C. S. Regazzoni and A. Teschioni, "A new approach to vector median filtering based on space filling curves," IEEE Trans. Image Processing, vol. 6, no. 7, pp. 1025-1037, 1997.

[29] M. Barni, V. Cappellini, and A. Mecocci, "Fast vector median filter based on Euclidean norm approximation," IEEE Signal Processing Letters, vol. 1, no. 6, pp. 92-94, 1994. 
[30] N. Nikolaidis and I. Pitas, "Nonlinear processing and analysis of angular signals," IEEE Trans. Signal Processing, vol. 46, no. 12, pp. 3181-3194, 1998.

[31] D. G. Karakos and P. E. Trahanias, "Generalized multichannel image-filtering structures," IEEE Trans. Image Processing, vol. 6, no. 7, pp. 1038-1045, 1997.

[32] M. Gabbouj and F. A. Cheikh, "Vector median-vector directional hybrid filter for color image restoration," in Proc. 8th European Signal Processing Conference (EUSIPCO '96), vol. 2, pp. 879-882, Trieste, Italy, September 1996.

[33] K. N. Plataniotis and A. N. Venetsanopoulos, "Vector processing," in Colour Image Processing, S. J. Sangwine, Ed., pp. 188-209, Chapman \& Hall, London, UK, May 1998.

[34] R. Lukac, "Adaptive color image filtering based on centerweighted vector directional filters," Multidimentional Systems and Signal Processing, vol. 15, no. 2, pp. 169-196, 2004.

[35] R. Lukac, B. Smolka, K. N. Plataniotis, and A. N. Venetsanopoulos, "Selection weighted vector directional filters," Computer Vision and Image Understanding, vol. 94, no. 1-3, pp. 140-167, 2004.

Rastislav Lukac received the M.S. (Ing.) and Ph.D. degrees in Telecommunications from the Technical University of Kosice, Slovak Republic in 1998 and 2001, respectively. From February 2001 to August 2002 he was an Assistant Professor at the Department of Electronics and Multimedia Communications at the Technical University of Kosice. Since August 2002 he has been a Researcher in Slovak Image Processing Center in Dobsina, Slovak Republic. From January 2003 to March 2003 he was a Postdoctoral Fellow at the Artificial Intelligence \& Information Analysis Lab at the Aristotle University of Thessaloniki, Greece. Since May 2003 he has been a Postdoctoral Fellow with the Edward S. Rogers Sr. Department of Electrical and Computer Engineering at the University of Toronto in Toronto, Canada. His research interests include digital camera image processing, microarray image processing, multimedia security, and nonlinear filtering and analysis techniques for color image \& video processing. Dr. Lukac is a Member of the IEEE Signal Processing Society. In 2003 he was awarded the NATO/NSERC Science Award.

Konstantinos N. Plataniotis received the B. Engineering degree in computer engineering from the Department of Computer Engineering and Informatics, University of Patras, Patras, Greece, in 1988, and the M.S. and Ph.D. degrees in electrical engineering from the Florida Institute of Technology (Florida Tech), Melbourne, Florida in 1992 and 1994, respectively. He was affiliated to the Computer Technology Institute (CTI),

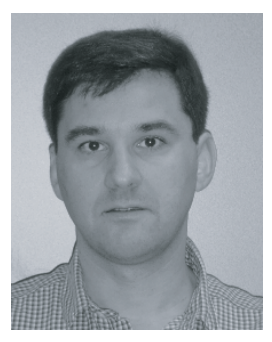
Patras, Greece, from 1989 to 1991. From August 1997 to June 1999, he was an Assistant Professor at the School of Computer Science, Ryerson University. He is currently an Assistant Professor at the Edward S. Rogers Sr. Department of Electrical \& Computer Engineering, where he researches and teaches adaptive systems and multimedia signal processing. Dr. Plataniotis is a Senior Member of IEEE, a past Member of the IEEE Technical Committee on Neural Networks for Signal Processing, and the Technical Cochair of the Canadian Conference on Electrical and Computer Engineering (CCECE) 2001 and CCECE 2004.
Bogdan Smolka received the Diploma in Physics degree from the Silesian University, Katowice, Poland, in 1986, and the Ph.D. degree in automatic control from the Department of Automatic Control, Silesian University of Technology, Gliwice, Poland. From 1986 to 1989, he was a Teaching Assistant at the Department of Biophysics, Silesian Medical University, Katowice, Poland. From 1992 to 1994, he worked as a Teach-

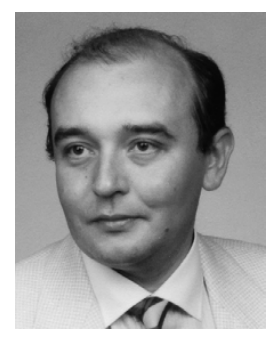
ing Assistant at the Technical University of Goeppingen, Germany. Since 1994, he has been with the Silesian University of Technology. In 1998, he was appointed as an Associate Professor at the Department of Automatic Control, Silesian University of Technology. Since 1999, he is also an Associate Researcher at Multimedia Laboratory, University of Toronto, Canada. His current research interests include low-level color image processing, human-computer interaction, wavelet-based methods of image compression, and the visual aspects of image quality.

Anastasios N. Venetsanopoulos received the B.S. degree in electrical and mechanical engineering from the National Technical University of Athens, Greece, in 1965 and the M.S., M.Phil., and Ph.D. degrees in electrical engineering from Yale University in 1966, 1968, and 1969, respectively. Since 1969, he has been a Professor at the Edward S. Rogers Sr. Department of Electrical and Computer Engineering, University

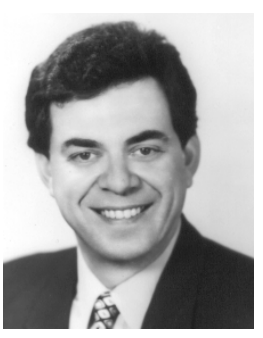
of Toronto. He is a contributor to 31 books, and has published over 740 papers in refereed journals and conference proceedings on digital signal and image processing and digital communications. Since July 2001, he has been the 12th Dean of the Faculty of Applied Science and Engineering, University of Toronto. Professor Venetsanopoulos was elected as a Fellow of the IEEE; he is also a Fellow of the EIC, and was awarded an Honorary Doctorate from the National Technical University of Athens in October 1994. In November 2000, he became a recipient of the Millennium Medal of IEEE. In April 2001, he became a Fellow of the Canadian Academy of Engineering. In 2003, he was the recipient of the highest award of the Canadian IEEE, the MacNaughton Award, and in 2003, he served as the Chair of the Council of Deans of Engineering of the Province of Ontario (CODE). 